TRANSACTIONS OF THE

AMERICAN MATHEMATICAL SOCIETY

Volume 182, August 1973

\title{
VISITATIONS OF RULED SUMS $\left(^{1}\right)$
}

BY

\section{LEONARD E. BAUM AND H. H. STRATTON}

\begin{abstract}
Let $\left\{X_{i}\right\}$ be a sequence of independent identically distributed random variables and for $D \subseteq I^{+}$let $S_{D}=\Sigma_{i \in D} X_{i}$. A rule ( ) is a mapping $I^{+} \rightarrow 2^{I^{+}}: \forall n|(n)|=n$ and $S_{()}=\left\{S_{(n)}\right\}$ is its associated ruled sum.

Ruled sums generalize ordinary sums $S_{n}$. Indeed, all a.e. results for $S_{n}$ can be investigated for $S_{(n)}$ frequently requiring different methods and sometimes yielding different conclusions. In a previous paper we studied strong laws of large numbers and the law of the iterated logarithm.

In this paper we study infinite visitation. Under suitable hypotheses on the basic distribution function $F$ of the $X_{i}$ we show that, for all rules ()$, S_{(n)}$ visits each integer infinitely often a.e. in the lattice case (or has all points of the real line as accumulation points in the nonlattice case). In fact we obtain a "rate of visitation." There follows extensions of the Pólya theorem on encounters in the plane and 3-space from random walks to the se ruled sums.

Some equivalence relations and partial orderings on rules are defined. For normal variables this leads to an extension of the previously mentioned result for ruled sums of the type of the iterated logarithm law.
\end{abstract}

I. Introduction and statement of visitation results. Let $\left\{X_{i}\right\}$ be a sequence of independent, identically distributed random variables (i.i.d.), and for $D \subseteq I^{+}$ ( $\equiv$ set of positive integers) let $S_{D} \equiv \Sigma_{i \in D} X_{i}$. Let $\mathbf{R} \equiv$ set of all functions ( ): $I^{+} \rightarrow 2^{I^{+}}$where $(n)$ contains exactly $n$ elements for each $n \in I^{+}$. ( ) $\in \mathbf{R}$ is called a rule and $S_{(n)}$ is called its ruled sum. Intuitively there are two rules that are in some sense the "extreme rules":

(1) $(n) \equiv\{1,2, \cdots, n\}$ which we denote by $n$,

(2) any rule ( ) that satisfies $(n) \cap(m)=\varnothing$ for $n \neq m$; we denote such a rule by \langle\rangle .

Certainly $n$ and $\langle n\rangle$ are at the top and bottom respectively in the two partial orderings given by

$$
() \leqq()^{\prime} \text { iff }\left|\bigcap_{i=1}^{m}\left(n_{i}\right)\right| \leq\left|\bigcap_{i=1}^{m}\left(n_{i}\right)^{\prime}\right| \text { for } n_{1}, \ldots, n_{m^{\prime}} m \in I^{+}
$$

Received by the editors November 23, 1971 and, in revised form, November 20, 1972. AMS (MOS) subject classifications (1970). Primary 60G50, 60F 15, 60F 20; Secondary $60 \mathrm{G} 15,60 \mathrm{~J} 15$.

Key words and phrases. Sums of independent variables, visitation infinitely often, zero one laws, vis itation rates, random walk, weakly dependent variables, Pólya meetings, Gaussian, ruled sums.

(1) This research was supported in part by NSF Grant GP 1922. 
and

$$
() \leq()^{\prime} \text { iff }\left|\left(n_{1}\right) \cap\left(n_{2}\right)\right| \leq\left|\left(n_{1}\right)^{\prime} \cap\left(n_{2}\right)^{\prime}\right| \text { for } n_{1}, n_{2} \in I^{+} \text {. }
$$

$(|A|$ means the cardinality of the set $A .$.$) \leqq is the natural ordering on rules since$ $\mathbf{R} \leqq \mathbf{R}^{\prime}$ and $\mathbf{R}^{\prime} \leqq \mathbf{R}$ iff $\mathbf{R} \cong \mathbf{R}^{\prime}$ under the natural equivalence relation:

$$
\begin{aligned}
& () \cong()^{\prime} \text { iff there exists a 1-1 map } \sigma \text { from } \\
& \bigcup_{n=1}^{\infty}(n) \text { onto } \bigcup_{n=1}^{\infty}(n)^{\prime} \text { such that } \bigcup_{i \in(n)}\{\sigma(i)\}=(n)^{\prime} \\
& \text { for } n \in I^{+} .
\end{aligned}
$$

(See Theorem 6 of $\$$ III.)

The weaker ordering $\leq$ is more natural when $X_{i}$ are normally distributed since a Gaussian sequence $S_{(n)}$ is determined by its covariance $E\left(S_{(n)} S_{(j)}\right)=$ $|(n) \cap(j)|$ assuming $X_{1}$ is $\pi(0,1)$. Moreover, the following two results of $\S_{3}$ help to confirm that the intuition and this ordering are in accord.

Proposition 2. For $X_{1} \Re(0,1),() \leq()^{\prime}$ iff $P\left[s_{(n)} \in(u, v) \mid s_{(j)} \in(u, v)\right] \leq$ $P\left[S_{(n)}, \epsilon(u, v) \mid S_{(j)}, \epsilon(u, v)\right]$ for all $n, j \in I^{+}$and $-\infty \leq u \leq v \leq \infty$.

Theorem 7. For $x_{1} \Re(0,1),() \leq()^{\prime}$ implies $\overline{\lim } S_{(n)}, / a_{n} \leq \overline{\lim } S_{(n)} / a_{n}$ for all sequences $\left\{a_{n}\right\}: a_{n} \rightarrow \infty$.

When $X_{1}$ is a normal variable, Theorem 7 extends the result

$$
\varlimsup \overline{\lim } S_{n} / a_{n} \leq \varlimsup \lim S_{(n)} / a_{n} \leq \varlimsup \lim S_{\langle n\rangle} / a_{n}
$$

obtained in [1] for symmetric $X_{1}$ and "well-behaved" sequences $\left\{a_{n}\right\}$.

Thus a conjecture like

(C) A theorem which holds for $n$ and \langle\rangle holds for all ( ) $\in R$

arises rather naturally. An unfortunate instance where $(\mathcal{C})$ fails is that although the tail sigma field of $\left\{S_{(n)}\right\}$ for ()$=n$ or \langle\rangle is trivial (the Hewitt-Savage theorem [3, p. 63]), it need not be trivial for more general rules. Indeed the tail event $\left[\left|S_{(2 n)}-S_{(2 n-1)}\right|<\epsilon\right.$ i.o.] will never be a $0-1$ event for certain rules if $1>P\left[\left|X_{1}\right|<\epsilon\right]>0$ (see $\left.\S 4\right)$. Nevertheless, one hopes that $(\mathcal{C})$ holds for certain classes of theorems. This paper is largely devoted to $(\mathcal{C})$ for the class of theorems dealing with the visitations and distribution of values of the sequence of ruled sums of a rule.

In the case of visitation theorems, we take $X_{1}$ to be a lattice random variable on $I$ ( $\equiv$ set of integers). Without loss of generality we can as sume that $X_{1}$ satisfies 
(w) $\underset{i, j \in I}{\operatorname{gcd}}\left\{|j-i|: P\left[X_{1}=j\right]>0\right.$ and $\left.P\left[X_{1}=i\right]>0\right\}=1 \quad[10$, p. 232].

Hence if $P\left[X_{1}<0\right]>0$ and $P\left[X_{1}>0\right]>0$ as we assume throughout then $\forall i \in I$, $\exists n(i) \in I^{+}: P\left[S_{n(i)}=i\right]>0$.

For $\left\{B_{n}\right\}$ a sequence of subsets of $I$,

$$
m_{n}\left(B_{n}\right) \equiv P\left[S_{n} \in B_{n}\right] \text { and } M_{n}\left(B_{n}\right) \equiv \sum_{i=1}^{n} m_{i}\left(B_{i}\right)+1 \text {. }
$$

If $B_{n}=\{a\}$, then we write $m_{n}(a)$ and $M_{n}(a)$ respectively. If $a=0$, then we suppress the $a^{\prime} s$ in the symbols altogether as we will do whenever $B_{n}$ is understood. $\phi(A)$ denotes the indicator function of the set $A, \hat{f}(\theta)$ the characteristic function of $X_{1}$.

The results in this paper on visitation theorems will now be discussed. In the following $K$ denotes differing positive constants and $(*)$ or $(* *)$ will be used for differing displayed items used only locally.

Theorem 1. Let ( ) $\in \mathbf{R}, E X^{+}=E X^{-}$, and let $M_{n} \vec{n}^{\infty}$.

(1) Let a $\in I$, and $g: R^{+}$(set of positive reals) ${ }^{n} \rightarrow R^{+}$, where $g(x) \uparrow \infty$ and its derivative $g^{\prime}(x)>0$ and $\downarrow$. If $g$ satisfies for some $\epsilon_{0} \in(0,1)$

$$
\begin{gathered}
\sum_{k=j}^{n} g^{\prime}\left(M_{k}(a)\right)\left(P\left[S_{(k)}=a \mid S_{(j)}=a\right]-P\left[S_{(k)}=a\right]\right) \\
\leq K\left(g^{\epsilon^{0}}\left(M_{n}(a)\right)\right) \quad \text { for all } 1 \leq j \leq n \in I^{+}
\end{gathered}
$$

then

$$
\lim _{n}\left(g\left(M_{n}(a)\right)\right)^{-1} \sum_{i=1}^{n} g^{\prime}\left(M_{i}(a)\right) \phi\left[S_{(i)}=a\right]=1 \text { a.e. }
$$

(2) Let $m_{n} \geq n^{-a}$ for all $n \in I^{+}$with $0<a<1$. If $\exists \epsilon<1 / 2: \forall a_{1}, a_{2} \in I$, $\forall 1 \leq j \leq n \in I^{+}$.

$$
\sum_{k=j}^{n} P\left[S_{(k)}=a_{1} \mid S_{(j)}=a_{2}\right]=O\left(M_{n}^{1+2 \epsilon}\right)
$$

then, for $a \in I$,

$$
P\left[\left|\sum_{k=1}^{n} \phi\left[S_{(k)}=0\right]-\sum_{k=1}^{n} \phi\left[S_{(k)}=a\right]\right|>M_{n}^{3 / 4+\epsilon / 2+\delta} \text { i.o. }\right]=0
$$

for all $\delta>0$.

(3) If ( $\dagger$ ) bolds for $g(x)=\lg x, m_{n} \geq n^{-\alpha}$ and ( $\dagger$ ) is satisfied, then 


$$
\sum_{k=1}^{n} \phi\left[S_{(k)}=0\right] / \sum_{k=1}^{n} \phi\left[S_{(k)}=a\right] \vec{n}^{1} \text { a.e. }
$$

Chung and Erdös [4] essentially showed that for ()$=n$ and $E X^{-}=E X^{+}$, ( $\dagger$ ) holds for $g(x)=\lg x$, and $\epsilon=0$ and $(\dagger \dagger)$ holds with $\epsilon=0$, i.e., they showed that, for all $a \in I, \lim _{n}\left(\lg \left(M_{n}\right)\right)^{-1} \sum_{k=1}^{n} \phi\left[s_{k}=a\right] / M_{k} \rightarrow 1$,

$$
P\left[\left|\sum_{k=1}^{n} \phi\left[s_{k}=a\right]-\sum_{k=1}^{n} \phi\left[s_{k}=0\right]\right|>M_{n}^{3 / 4}+\delta \text { i.o. }\right]=0
$$

for all $\delta>0$, and $\Sigma_{k=1}^{n} \phi\left[S_{k}=a\right] / \Sigma_{k=1}^{n} \phi\left[S_{k}=0\right] \vec{n} 1$ a.e. The proofs for (1)(3) of Theorem 1 are adaptations of the proofs for ()$=n$ that appear in [4]. The main difference in proof arises in finding a replacement for their repeated appeal to the Markov property of $S_{n}$ which is, of course, lacking for general rules.

We note that since $S_{\langle n\rangle}$ is a sequence of independent random variables, $(\dagger)$ and $(\dagger \dagger)$ always hold. Thus (1)-(3) of Theorem 1 are candidates for $(\mathcal{C})$. In this direction, we will show

Proposition 1. If $X_{1}$ is symmetric, then for ( ) $\in \mathbf{R}$,

(1) $(\dagger)$ bolds for all $a \in I$ and $g(x)$ which satisfies, for some $1>\epsilon_{0}>0$, $x g^{\prime}(x)=O\left(g^{\epsilon} 0(x)\right)$, e.g., $g(x)=O\left(\lg ^{k}(x)\right)$ for $k \in I$.

(2) (††) bolds.

Because $P\left[S_{n+1}=a\right] \geq m_{1}\left(a-a^{\prime}\right) P\left[S_{n}=a^{\prime}\right]$, we see for $M_{n} \rightarrow \infty$

$$
M_{n}(a) / M_{n}\left(a^{\prime}\right)=O(1) \text { for } a, a^{\prime} \in I \text {. }
$$

(Note $(\rho)$ also holds if $S_{n}$ were the sum of $n$ i.i.d. $k$-dimensional random vectors.)

If $X_{1}$ is in the domain of attraction of a stable law of exponent $1 / a>1$ (e.g., for $E X_{1}^{2}<\infty, a=1 / 2$ ), then $P\left[S_{n}=0\right] \sim K_{n}^{-a}$. Thus $(\rho)$ combined with Theorem 1 and Proposition 1 yields

Corollary 1. Let $X_{1}$ be symmetric and $M_{n} \rightarrow \infty$. For ()$\in \mathbf{R}$,

(a) $\left(\lg M_{n}(a)\right)^{-1} \sum_{k=1}^{n} \phi\left[S_{(k)}=a\right] / M_{k}(a) \frac{n}{n} 1$ a.e. for all $a \in I$.

(b) If $X_{1}$ is, moreover, in the domain of attraction of a stable law with exponent $>1$, then for all $a \in I$

$$
\sum_{k=1}^{n} \phi\left[S_{(k)}=a\right] / \sum_{k=1}^{n} \phi\left[S_{(k)}=0\right] \underset{n}{\rightarrow} \text { a.e. }
$$

Now $(\rho)$, the Borel-Cantelli lemma, (a) of Corollary 1 and the fact that

$$
\left.\left(\lg M_{n}(a)\right)^{-1} \sum_{k=1}^{n} \phi\left[S_{(k)}=a\right] / M_{k}(a)=\mathcal{Q}\left(\lg M_{n}(a)\right)^{-1} \sum_{k=1}^{n} \phi\left[S_{(k)}=a\right]\right)
$$

gives 
Corollary 2. For $X_{1}$ symmetric, $a \in I$ and ()$\in \mathbf{R}$

$$
P\left[S_{(n)}=a \text { i.o. }\right]=\frac{1}{0} \text { iff } \sum_{n=1}^{\infty} P\left[S_{n}=0\right] \underset{<\infty}{=\infty} \text { iff } \int_{-\pi}^{\pi} \frac{d \theta}{1-f(\theta)}<\infty .
$$

(The characteristic function part of Corollary 2 is well known and is proved easily from $(C 2)$ and $(C 5)$ in the first part of $\$$ II. Corollary 2 has its genesis in Chung and Fuchs [5] for ()$=n$ and general $X_{1}$.)

Theorem 2. Let $X_{1}$ be a lattice random variable. Then the following nine conditions are equivalent.

(1) $\int_{-\pi}^{\pi}(1-|\hat{f}(\theta)|)^{-1} d \theta=\infty$.

(2) $\Sigma_{n} \Sigma_{a} P^{2}\left[S_{n}=a\right]=\infty$.

(3) $\exists d_{n} \in I: \sum P\left[S_{n}=d_{n}\right]=\infty$.

(4a) $\forall() \in \mathbf{R}: \sum m_{n}\left(S_{(n)}(\omega)\right)=\infty$ a.s.

(4b) $\exists\left(\right.$ ) $\in \mathbf{R}: P\left[\Sigma m_{n}\left(S_{(n)}(\omega)\right)=\infty\right]>0$.

(5a) $\forall() \in \mathbf{R} \exists d_{n} \in I: \forall a \in \mathbf{R} P\left[S_{(n)}-d_{n}=a\right.$ i.o. $]=1$.

(5b) $\exists\left(\right.$ ) $\in \mathbf{R}, d_{n} \in I$ and $a \in \mathbf{R}: P\left[S_{(n)}-d_{n}=a i .0.\right]>0$.

(6a) $\forall()_{1},()_{2} \in \mathbf{R}:(n)_{1} \cap(m)_{2}=\varnothing \forall n, m \in I^{+} P\left[S_{(n)_{1}}=S_{(n)_{2}}+a\right.$ i.o. $]=$ $1, \forall a \in I$.

(6b) $\exists()_{1},()_{2} \in \mathbf{R}:(n)_{1} \cap(m)_{2}=\varnothing \forall n, m \in I^{+}$; and $\exists a \in I: P\left[S_{(n)_{1}}=\right.$ $S_{(n)_{2}}+a$ i.o. $]>0$.

These nine equivalent conditions above generalize the notion of recurrence to essential recurrence in the spirit of $\left[10\right.$, p. 250]. Even for $S_{n}$, this is a true extension of recurrence. In particular, if $E\left|X_{1}\right|<\infty$ and $E X_{1} \neq 0$ then $P\left[S_{n}=\right.$ $a$ i.o. $]=0$ for all $a$, while $P\left[S_{n}-n E X_{1}=a\right.$ i.o. $]=1$ for all $a[3, \mathrm{p} .56]$. Of course, if $X_{1}$ is symmetric, then (1) is equivalent to $\int_{-\pi}^{\pi}(1-\hat{f}(\theta))^{-1} d \theta=\infty$, i.e., essential recurrence becomes ordinary recurrence.

Theorem 2 seems fairly complete since condition (1) is a hypothesis on the basic datum, the distribution of $X_{1}$, and moreover, no extraneous hypothesis is needed. The same is true for Theorem 3.

Theorem 3. Let $\left(X_{i}\right)$ be i.i.d. lattice random variables. Let ()$_{1},()_{2},()_{3}$, ()$_{4} \in \mathbf{R}$ for which $(n)_{i} \cap(m)_{j}=\varnothing$ for $i \neq j=1,2,3,4$, and $n, m \in I^{+}$. The following five conditions are equivalent.

(1) $\int_{-\pi}^{\pi} \int(1-|\hat{f}(\theta) f(\phi)|)^{-1} d \theta d \phi=\infty$.

(2) $\Sigma_{n}\left(\Sigma_{a} P^{2}\left[S_{n}=a\right]\right)^{2}=\infty$.

(3) $P\left[\left(S_{(n)_{1}}, S_{(n)_{2}}\right)=\left(S_{(n)_{3}}, S_{(n)_{4}}\right)\right.$ i.o. $]=1$.

(4) $\Sigma_{n} \Sigma_{a} P^{3}\left[S_{n}=a\right]=\infty$.

(5) $P\left[S_{(n)_{1}}=S_{(n)_{2}}=S_{(n)_{3}}\right.$ i.o. $]=1$. 
(3) and (5) of Theorem 3 are, as was (6) of Theorem 2, extensions of Pólyatype theorems; e.g., (6) of Theorem 2 is an extension of two independent particles, each of which is performing a random walk in one dimension, meeting infinitely often (Feller [6, vol. 1, p. 329]).

Of course, one could consider similar questions for nonlattice random variables. For $E X_{1}^{2}<\infty$, an application of a local limit theorem in the proof of Theorem 2 will yield Theorem 2 with points of infinite visitation replaced by accumulation points. An example of how one is to carry out these suggested steps is given in the proof of the following:

Theorem 4. Let $E X_{1}^{2}<\infty$ and let $\left\{a_{n}\right\}$ be a nondecreasing sequence of positive real numbers with $a_{n}=o\left(n^{1 / 2}\right)$. Then the following are equivalent.

(1) $E X=0$.

(2) For some $b \in$ Reals and some ( ) $\in \mathbf{R}, P[b$ is an accumulation point of $\left.S_{(n)} / a_{n}\right]>0$.

(3) For all $b \in$ Reals and all ()$\in \mathbf{R}, P[b$ is an accumulation point of $\left.S_{(n)} / a_{n}\right]=1$.

(4) $\Sigma_{n} P\left[\left|S_{n} / a_{n}\right|<\epsilon\right]=\infty$ for all $\epsilon>0$.

This result was obtained for ()$=n$ and $a_{n}=n-a, 0<a<1 / 2$, without the assumption $E X_{1}^{2}<\infty$ by Kesten [8, Theorem 4, p. 1173]. We note here that our proof of Theorem 4 actually shows that $(3) \Rightarrow$ (1) under the weaker assumption that $a_{n}=o(n)$. However Kesten shows that one cannot in general let $a_{n}=n$ and have $(1) \Rightarrow(3)\left[8\right.$, Theorem 7 , p. 1196]. Finally we see that by taking $a_{n}=1$ for all $n$, we obtain Corollary 2 with the following modifications. Replace the hypothesis $X_{1}$ symmetric with $E\left(X_{1}\right)^{2}<\infty$ and insert Real part in front of the integral.

Theorem 5. Let $a>0$ be such that it is not a rational multiple of the span of $X_{1}$ when $X_{1}$ is a lattice variable. For ()$\in \mathbf{R}, S_{(n)}$ is equidistributed $\bmod [0, a)$.

This theorem generalizes a result of Robbins $[16]$ for ()$\equiv n$. Theorem 5 requires no extra distribution hypotheses and has a simple proof. Corollary 1 with its subsidiary hypotheses and a more difficult proof yields asymptotically equal number of visits to any pair of lattice points whereas Theorem 5 concludes only that the total number of visits in time $N$ to all points in $\bigcup_{i \in I}\{(b, c)+i a\} \sim$ $N(c-b) / a$ where $b<c<b+a$.

This completes our statements of our main visitation results. Their proofs will be given in $\S$ II. In $\S$ III we deal with two equivalence relations, and two partial orderings on rules, and prove related results some of which were stated earlier in this introduction. In $\S \mathrm{IV}$, which consists mainly of counterexamples, 
we show some of the extent to which triviality of the tail $\sigma$-field fails for ruled sums. In $\$ \mathrm{~V}$ we give extensions of some of our main visitation results to Gaussian processes more general than ruled sums of independent normal $(0,1)$ variables.

II. Proofs of visitation results. We first gather together for easy reference a collection of basic results that will be essential to the proofs of Theorems 1-4 and Corollaries 1, 2.

Lemma 0. Let $\left\{Y_{n}\right\}$ be an arbitrary sequence of random variables with $E\left(Y_{n}^{2}\right) \leq c_{n}$ for all $n \in I^{+}$. If there exists a subsequence $\{n(k)\}$ of $I^{+}$with

(a) $\Sigma_{k} c_{n(k)}<\infty$,

(b) $\max _{n(k) \leq n \leq n(k+1)}\left|Y_{n}-Y_{n(k)}\right| \vec{k} 0$ a.e.

then $Y_{n} \rightarrow 0$ a.e.

Proof. Standard use of the Borel-Cantelli lemma and the Markov inequality.

In the rest of this collection of basic results we let $A, A^{\prime}, B, B^{\prime} \subseteq I^{+}$with $C \equiv A \cap B, C^{\prime} \equiv A^{\prime} \cap B^{\prime}$ and $a \equiv|A|, a^{\prime} \equiv\left|A^{\prime}\right|$, etc. We assume without loss of generality that $a \geq b$ and $a^{\prime} \geq b^{\prime}$. For $D \subseteq I^{+}, S_{D} \equiv \Sigma_{i \in D} X_{i}$. Further, we let $d, f, b, i, l \in I$ and $k, m, n \in I^{+}$.

Symmetrization. An often used technique in probability theory is the symmetrization of a random variable $Y[12$, p. 245]. One considers a random variable $Y^{\prime}$ independent of but identically distributed to $Y$, and defines the symmetric random variable $Y^{s}=Y-Y^{\prime}$, to be the symmetrization of $Y$. Similarly, if $Y_{1}$, $Y_{2}, \cdots$ are random variables then $S_{k}^{s} \equiv \sum_{i=1}^{k} Y_{i}^{s}$ where the $Y_{i}^{\prime}$ are independent of each other and of the $\left\{Y_{j}\right\}$ sequence. If $\hat{f}(\theta)$ is the characteristic function of $Y$ then $|\hat{f}(\theta)|^{2}$ is the characteristic function of $Y^{s}$.

Needed results about the characteristic function $\hat{f}(\theta)$ of a symmetric random variable $X$. If $X$ is the symmetrization of a random variable $Y$, then clearly $\hat{f}(\theta) \geq 0$ for all $\theta \in(-\pi, \pi]$, but this does' not hold for a general symmetric $X$. Thus we define $\epsilon_{0} \equiv \max \{\epsilon \epsilon(0, \pi]: \hat{f}(\theta) \geq 0$ for $|\theta| \leq \epsilon\}$ and $s_{0} \equiv \sup \{|\hat{f}(\theta)|: \theta \epsilon$ $\left.\left(-\pi,-\epsilon_{0}\right) \cup\left(\epsilon_{0}, \pi\right)\right\}$ where $s_{0}=0$ if $\epsilon_{0}=\pi$.

That $\epsilon_{0}$ and $s_{0}$ are well defined is contained in

(C1) $\hat{f}(\theta)$ is real valued, continuous at $0, \hat{f}(0)=1$ (i.e., $\epsilon_{0}>0$ ) and $s_{0}<1$ (because $X_{1}$ satisfies $(\omega)$ ).

By the inversion formula for characteristic functions:

(C2) $P\left[S_{n}=l\right]=(2 \pi)^{-1} \int_{-\pi}^{\pi} \cos (l \theta) \hat{f}^{n}(\theta) d \theta$.

By the definition of $s_{0}$, we see $(2 \pi)^{-1} \int_{-\pi}^{-\epsilon_{0}}+\int_{\epsilon_{0}}^{\pi}\left|\hat{f}^{n}(\theta)\right| d \theta \leq s_{0}^{n}$ and so

$$
P\left[S_{n}=0\right]-P\left[S_{n}=l\right] \geq(2 \pi)^{-1} \int_{-\epsilon_{0}}^{\epsilon_{0}}(1-\cos (l \theta)) \hat{f}^{n}(\theta) d \theta-2 s_{0}^{n} \geq-2 s_{0}^{n} .
$$

Thus we have 

$P\left[s_{n}=l\right]$.

(C3) $\left|P\left[S_{n}=l\right]-(2 \pi)^{-1} \int_{-\epsilon_{0}}^{\epsilon_{0}} \cos (l \theta) \hat{f}^{n}(\theta) d \theta\right|<s_{0}^{n}$ and $P\left[S_{n}=0\right]+2 s_{0}^{n} \geq$

Let $\mu$ be the counting measure on $I$, then for $f, g \in \mathscr{L}^{2}(I, \mu)$

(C4) (Plancherel's theorem)

$$
\sum_{b} f(b) g(b)=\frac{1}{2 \pi} \int_{-\pi}^{\pi} \hat{f}(\theta) \overline{\hat{g}(\theta)} d \theta
$$

where $\hat{f}$ and $\hat{g}$ are the Fourier transforms of $f$ and $g$.

Needed results from [4]. Let $X_{1}$ be a lattice variable satisfying $(\omega)$ and $E\left(X_{1}^{+}\right)=E\left(X_{1}^{-}\right)$(where $\infty$ is allowed).

(CE1) $P\left[S_{n}=b\right] / P\left[S_{n-m}=l\right] \vec{n} 1$ (Theorem 4 of [4]).

(CE2) For $r>0, \exists$ a constant $K$ depending on $r, \epsilon$, and $b-l$ but not on b or $n: \forall \epsilon>0$

$$
P\left[S_{n}=b\right]-P\left[S_{n}=l\right] \leq K n^{-1 / 2+\epsilon} P\left[S_{n}=b\right]+n^{-r} .
$$

In Theorem 3.2 of [4] Chung and Erdös do not claim $K$ depends only on $b-l$ but their proof reveals this is so.

Needed results about ruled sums. We first state a corollary of Hölder's inequality:

(R0) Let $\left\{a_{n}\right\}$ be a sequence of positive numbers and let $\{i(l)\}$ and $\{j(l)\}$ be two 1-1 maps of 1 onto $I$. Then

(a) $\Sigma_{l} a(l) a(i(l)) \leqq \Sigma_{l}(a(l))^{2}$,

(b) $\Sigma_{l} a(l) a(i(l)) a(j(l)) \leqq \Sigma_{l}(a(l))^{3}$.

(R1) If $a=b=a^{\prime}=b^{\prime}$ and $c \geq c^{\prime}$ then $P\left[S_{A}=l, S_{B}=l\right] \geq P\left[S_{A},=\right.$ $\left.l, S_{B},=l\right]$.

(In particular, $P\left[s_{A}=l, s_{B}=l\right] \geq P^{2}\left[s_{a}=l\right]$.)

Proof. Without loss of generality we assume $c=c^{\prime}+1$.

$$
\begin{aligned}
P\left[s_{A}\right. & \left.=l, s_{B}=l\right]=\sum_{b} P^{2}\left[s_{|A|-|C|}=l-b\right] P\left[s_{C}=b\right] \\
& =\sum_{b} \sum_{d} P^{2}\left[s_{a-c}=l-b\right] P\left[X_{1}=b-d\right] P\left[s_{c^{\prime}}=d\right] \\
& =\sum_{d} P\left[s_{c^{\prime}}=d\right] \sum_{b} P^{2}\left[s_{a-c}=l-b\right] P\left[X_{1}=b-d\right] \\
& \geq \sum_{d} P\left[s_{c^{\prime}}=d\right]\left[\sum_{b} P\left[s_{a-c}=l-b\right] P\left[X_{1}=b-d\right]\right]^{2} \\
& =\sum_{d} P\left[s_{c^{\prime}}=d\right] P^{2}\left[s_{a-c^{\prime}}=l-d\right]=P\left[s_{A^{\prime}}=l, s_{B^{\prime}}=l\right]
\end{aligned}
$$

where the inequality is the Schwarz inequality with respect to the probability 
measure $\lambda_{d}$ on $I$ given by $\lambda_{d}(\{i\}) \equiv P\left[X_{1}=i-d\right]$ for $i \in I$.

(R2) (a) Let $\mathcal{G}$ be an event independent of $\left\{X_{i}\right\}, i \in A^{\prime} \subseteq A$. If $X_{i}$ is sym. metric

$$
P\left[s_{A}=d, \mathfrak{Q}\right] \leq\left(P\left[s_{a^{\prime}}=0\right]+2 s_{0}^{a^{\prime}}\right) P[\mathfrak{Q}]
$$

(b) Let $\mathfrak{Q}$ be an event independent of $\left\{X_{i}\right\}, i \in A^{\prime} \cup B^{\prime}$ where $A^{\prime} \subseteq A$, $B^{\prime} \subseteq B, A \cap B=\varnothing$ and $a^{\prime}=b^{\prime}$. Then

$$
P\left[s_{A}-s_{B}=d, \mathfrak{Q}\right] \leq P\left[s_{a^{\prime}}^{s}=0\right] P[\mathscr{Q}] \text {. }
$$

(c) Let $\mathbb{Q}$ be an event independent of $\left\{X_{i}\right\}, i \in A^{\prime} \cup B^{\prime} \cup C^{\prime}$, where $A^{\prime}$ $\subseteq A, B^{\prime} \subseteq B, C^{\prime} \subseteq C, A, B$ and $C$ are pairwise disjoint and $a^{\prime}=b^{\prime}=c^{\prime}$. Then $P\left[s_{A}-s_{C}=0, s_{B}-s_{C}=0, \mathfrak{Q}\right] \leq P\left[s_{A^{\prime}}-s_{C^{\prime}}=0, s_{B^{\prime}}-s_{C^{\prime}}=0\right] P[\mathfrak{Q}]$.

Proof. (a) By (C3)

$$
\begin{aligned}
P\left[S_{A}=d, \mathfrak{Q}\right] & =\sum_{b} P\left[S_{A^{\prime}}=d-b\right] P\left[S_{A-A^{\prime}}=b, \mathfrak{Q}\right] \\
& \leq\left(P\left[S_{a^{\prime}}=0\right]+2 s_{0}^{a^{\prime}}\right) P[\mathfrak{Q}] .
\end{aligned}
$$

(b) Because $a^{\prime}=b^{\prime}$ and $A \cap B=\varnothing$, we see that $S_{A},-S_{B^{\prime}}$ is the sum of $a^{\prime}$ independent symmetrizations of $X_{1}$. Hence by hypothesis and (C3)

$$
\begin{aligned}
P\left[s_{A}-S_{B}=d, \mathbb{Q}\right] & =\sum_{b} P\left[s_{a^{\prime}}^{s}=d-b\right] P\left[s_{\dot{A}}-S_{B}-\left(s_{A^{\prime}}-S_{B^{\prime}}\right)=b, \mathbb{Q}\right] \\
& \leq P\left[s_{a^{\prime}}^{s}=0\right] P[\mathfrak{Q}]
\end{aligned}
$$

(c)

$$
\begin{aligned}
& P\left[s_{A}-S_{C}=0, s_{B}-S_{C}=0, \mathfrak{Q}\right] \\
& =\sum_{g, b} P\left[S_{A^{\prime}}-S_{C^{\prime}}=g, S_{B^{\prime}}-S_{C^{\prime}}=b\right] \\
& \text { - } P\left[S_{A}-S_{C}-\left(S_{A^{\prime}}-S_{C^{\prime}}\right)=-g, S_{B}-S_{C}-\left(S_{B^{\prime}}-S_{C^{\prime}}\right)=-b, \mathbb{Q}\right] \text {. } \\
& \leq \max _{g, b} P\left[S_{A^{\prime}}-S_{C^{\prime}}=g, S_{B^{\prime}}-S_{C^{\prime}}=b\right] P[\mathscr{Q}] \text {. }
\end{aligned}
$$

Since

$$
P\left[S_{A^{\prime}}-S_{C^{\prime}}=g, S_{B^{\prime}}-S_{C^{\prime}}=b\right]=\sum_{i} P\left[S_{C^{\prime}}=i\right] P\left[S_{A^{\prime}}=g+i\right] P\left[S_{B^{\prime}}=b+i\right],
$$

(R2c) now follows from the hypothesis $a^{\prime}=b^{\prime}=c^{\prime}$ and the case (b) of (R0).

(R3) Let $r>0$ and $E X_{1}^{+}=E X_{1}^{-} \cdot \exists$ a constant $K$ depending only on $r$ and $l-d: \forall \epsilon>0$ 


$$
\begin{aligned}
& P\left[s_{A}=l, s_{B}=b\right]-P\left[s_{A}=d, s_{B}=b\right] \\
& \quad \leq K(a-c)^{-1 / 2+\epsilon} P\left[s_{A}=l, s_{B}=b\right]+(a-c)^{-r} P\left[s_{B}=b\right] .
\end{aligned}
$$

Proof. Recalling that in (CE2) the constant $K$ depends only on the difference of the values, we have

$$
\begin{aligned}
P\left[S_{A}\right. & \left.=l, s_{B}=b\right]-P\left[s_{A}=d, s_{B}=b\right] \\
& =\sum_{f}\left(P\left[s_{a-c}=l-f\right]-P\left[s_{a-c}=d-f\right]\right) P\left[S_{C}=f, S_{B}=b\right] \\
& \leq \sum_{f}\left(K(a-c)^{-1 / 2+\epsilon} P\left[S_{a-c}=l-f\right]+(a-c)^{-r}\right) P\left[S_{C}=f, s_{B}=b\right] \\
& \leq K(a-c)^{-1 / 2+\epsilon} P\left[s_{A}=l, s_{B}=b\right]+(a-c)^{-r} P\left[S_{B}=b\right] .
\end{aligned}
$$

This ends our list of basic results, and we begin to prove the visitation results; (1) of Theorem 1 is actually a special case of the following more general result:

Lemma 1. Let $\left\{Z_{n}\right\}$ be a sequence of d dimensional lattice random vectors, $\left\{B_{n}\right\}$ be a sequence of subsets of $I^{d}$, and $g: R^{+} \rightarrow R^{+}$with $g(x) \uparrow_{\infty}$ and $g(x)$ positive and $\downarrow$. If there is an $\epsilon \in(0,1)$ so that

$$
\sum_{k=j}^{n} g^{\prime}\left(M_{k}\right)\left(P\left[Z_{k} \in B_{k} \mid Z_{j} \in B_{j}\right]-P\left[Z_{k} \in B_{k}\right]\right) \leq K\left(g^{\epsilon}\left(M_{n}\right)\right)
$$

for all $j, n \in I^{+}$and if $M_{n} \rightarrow \infty$, then

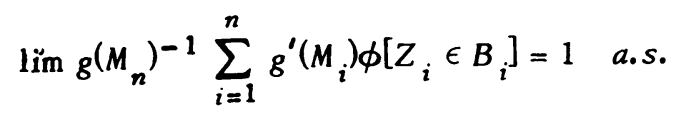

Furtbermore, if

$$
\overline{\lim }\left(1 / g\left(M_{n}\right)\right) \sum_{k=j}^{n} g^{\prime}\left(M_{k}\right) P\left[Z_{k} \in B_{k} \mid Z_{j} \in B_{j}\right]-P\left(\left[Z_{k} \in B_{k}\right]\right) \leq 0
$$

then

$$
P\left[Z_{j} \in B_{j} i_{\bullet} o_{0}\right]=1
$$

Proof. Let $L(n) \equiv \sum_{i=1}^{n} g^{\prime}\left(M_{i}\right) \phi\left[Z_{i} \in B_{i}\right]$. Because $g^{\prime}(x)$ is nonincreasing, we may use the standard approximation of sequences by the Riemann integral to obtain $E(L(n))=O(1)+g\left(M_{n}\right)$, i.e., since $g(x) \uparrow \infty$ and $M_{n} \uparrow \infty, E(L(n)) \sim g\left(M_{n}\right)$.

Letting $V_{n} \equiv E\left[\left(g\left(M_{n}\right)\right)^{-1} L(n)-1\right]^{2}$, we have 


$$
\begin{aligned}
g^{2}\left(M_{n}\right) V_{n} \leq & O\left(g\left(M_{n}\right)\right) \\
& +2 \sum_{k>j=1}^{n} g^{\prime}\left(M_{j}\right) g^{\prime}\left(M_{k}\right)\left(P\left[Z_{k} \in B_{k}, Z_{j} \in B_{j}\right]-m_{k} m_{j}\right) \\
= & O\left(g\left(M_{n}\right)\right) \\
& +2 \sum_{j=1}^{n} g^{\prime}\left(M_{j}\right) m_{j} \sum_{k=j+1}^{n} g^{\prime}\left(M_{k}\right)\left(P\left[Z_{k} \in B_{k} \mid Z_{j} \in B_{j}\right]-m_{k}\right) \\
= & O\left(g^{1+\epsilon}\left(M_{n}\right)\right) .
\end{aligned}
$$

Thus $V_{n}=O\left(g^{-1+\epsilon}\left(M_{n}\right)\right)$.

Proof of (1). We take $Y_{n} \equiv\left(g\left(M_{n}\right)\right)^{-1} L(n)-1, c_{n} \equiv\left(g\left(M_{n}\right)\right)^{-1+\epsilon}$ and $n(k)$ so that $g\left(M_{n(k)}\right)^{1-\epsilon} \sim k^{2}$ in Lemma 0 . Then $\Sigma_{k} c_{n(k)}<\infty$ is clear and so $Y_{n(k)}$ $\vec{k} 0$ a.e. But the choice of $n(k)$ gives $g\left(M_{n(k)}\right) / g\left(M_{n(k+1)}\right) \vec{k} 1$ and so

$$
\begin{aligned}
\max _{n(k) \leq n \leqq n(k+1)}\left|Y_{n}-Y_{n(k)}\right| \leq & \left(g\left(M_{n(k)}\right)\right)^{-1}\left(L_{n(k+1)}-L_{n(k)}\right) \\
& +L_{n(k)}\left(g\left(M_{n(k+1)}\right)-g\left(M_{n(k)}\right)\right)\left(g\left(M_{n(k)}\right)\right)^{-2} \\
& 0 \text { a.e. }
\end{aligned}
$$

Thus an application of Lemma 0 establishes (1).

Proof of (2). We have

$$
\begin{aligned}
V_{n} \leq & O\left(g\left(M_{n}\right)^{-1}\right) \\
& +\left(\frac{1}{g\left(M_{n}\right)} \sum_{j=1}^{n} g^{\prime}\left(M_{j}\right) m_{j}\right) \frac{1}{g\left(M_{n}\right)} \sum_{k=j+1}^{n} g^{\prime}\left(M_{k}\right)\left(P\left[Z_{k} \in B_{k} \mid Z_{j} \in B\right]-m_{k}\right) \rightarrow 0 .
\end{aligned}
$$

Choose $n(k)$ so that $V_{n(k)} \leq k^{-2}$. Then $\left(g\left(M_{n(k)}\right)\right)^{-1} L(n(k))-1=\vec{k}=0$ a.e. by the Borel-Cantelli lemma. But $g(x) \uparrow_{\infty}$ and so continuing as in the remarks preceding Corollary 2 in $\$ 1$, the proof of Lemma 1 is completed.

In this paper we are primarily interested, as in (1) of Theorem 1, in the case where $B_{n} \equiv\{a\}$ for all $n$ and some $a \in I^{k}$. (1) of Proposition 1 is a special case of

Lemma 2. Let $\left\{Z_{k}\right\}$ be a sequence of random variables and $g \in R^{+} \rightarrow R^{+}$ satisfy $g^{\prime}>0, g^{\prime} \downarrow$ and $x g^{\prime}(x)=O\left(g^{\epsilon} 0(x)\right)$ for some $\epsilon_{0} \in(0,1)$.

Let $M_{n} \rightarrow \infty$. Assume

(a) $\exists c>0$ and $v<1: \forall j, k \in I^{+}$with $j \leq k, P\left[Z_{k}=a \mid Z_{j}=a\right] \leq$ $P\left[Z_{k-j}=0\right]+c v^{k-j}$. 
(b) There is a sequence of i.i.d. random variables $\left\{Y_{j}\right\}$ satisfying $(\omega)$ with $E\left(Y_{1}\right)$ such that $P\left[Z_{k}=0\right]=P\left[\sum_{j=1}^{k} Y_{j}=0\right]$ for all $k$.

Then $\left(\dagger^{\prime}\right)$ bolds for $B_{n}=\{a\}$.

If the $Z_{k}$ are $d$-dimensional random vectors and $a$ is the $d$-dimensional 0 vector, then $\left(t^{\prime}\right)$ holds under the weaker hypothesis obtained by deleting (b).

Proof. By hypothesis (a) and the fact that $g^{\prime}(x)$ is bounded,

$$
\begin{aligned}
Q_{n} & \equiv \sum_{k=j+1}^{n} g^{\prime}\left(M_{k}\right)\left(P\left[Z_{k}=a \mid Z_{j}=a\right]-m_{k}\right) \\
& \leq \sum_{k=1}^{n-j} g^{\prime}\left(M_{k+j}\right) m_{k}(0)-\sum_{k=j+1}^{n} g^{\prime}\left(M_{k}\right) m_{k}+O(1)
\end{aligned}
$$

By the hypothesis on $g(x)$, hypothesis (b) and $(\rho)$ (i.e., $M_{n}(a) / M_{n}\left(a^{\prime}\right)=O(1)$ ) we see

$$
\sum_{k=1}^{j} g^{\prime}\left(M_{k+j}\right) m_{k}(0) \leq g^{\prime}\left(M_{j}\right) M_{j}(0)=O\left(g^{\epsilon}\left(M_{j}\right)\right) .
$$

Now let $\left\{b_{k}\right\}$ be a sequence in $R^{+}$with $b_{k} \leq 1$ for all $k$, let $r>\delta>0$, then by Hölder's inequality,

(H)

$$
\begin{aligned}
\sum_{k=1}^{n} k^{-r} b_{k} & \leq\left(\sum_{k=1}^{n} k^{-r /(r-\delta)}\right)^{r-\delta}\left(\sum_{k=1}^{n} b_{k}^{1 /(1-r+\delta)}\right)^{1-r+\delta} \\
& =O\left(\left(\sum_{k=1}^{n} b_{k}\right)^{1-r+\delta}\right) .
\end{aligned}
$$

We then have

$$
\begin{aligned}
& \sum_{k=j+1}^{n-j} g^{\prime}\left(M_{k+j}\right) m_{k}(0)-\sum_{k=j+1}^{n} g^{\prime}\left(M_{k}\right) m_{k} \\
& \leq \sum_{k=j+1}^{n} g^{\prime}\left(M_{k}\right)\left(m_{k}(0)-m_{k}\right) \text { since } g^{\prime}(x) \downarrow \\
& \leq A \sum_{k=j+1}^{n} g^{\prime}\left(M_{k}\right) m_{k}(0) k^{-1 / 2+\epsilon^{\prime}} \quad \begin{array}{l}
\text { for any } \epsilon^{\prime}>0 \text { by (CE2) } \\
\text { and hypothesis (b) }
\end{array} \\
& =O\left(\sum_{k=j+1}^{n} g^{\prime}\left(M_{k}\right) m_{k}(0)\right)^{1 / 2+\epsilon^{\prime \prime}} \text { for any } \epsilon^{\prime \prime}>\epsilon^{\prime} \text { by }(\mathrm{H}) \text {, } \\
& \leq O\left(g^{1 / 2+\epsilon^{\prime \prime}}\left(M_{n}\right)\right) \text {. }
\end{aligned}
$$


For the last in equality we have used the standard discrete approximation to the Riemann integral and the fact $g^{\prime}$ is nonincreasing. In the application of $(\mathrm{H})$ we have used the fact that $g^{\prime}$ is bounded and $m_{k}(0) \rightarrow 0$ so for all large $k$ $g^{\prime}\left(M_{k}\right) m_{k}(0) \leq 1$.

Since $\epsilon^{\prime}$ may be arbitrarily close to 0 and $\epsilon^{\prime \prime}$ arbitrarily close to $\epsilon^{\prime}$, any $\epsilon>\max \left(1 / 2, \epsilon_{0}\right)$ suffices for $\left(\dagger^{\prime}\right)$.

Finally, when the $Z_{k}$ are $d$-dimensional but $a$ is the $d$-dimensional 0 vector, the proof becomes much simpler since only the hypothesis on $g$ implies $(*)$ and that the expression $(* *)$ is $\leq 0$.

Proof of (2) of Theorem 1. Let $D(l, n) \equiv \sum_{k=l}^{n} \phi\left[S_{(k)}=a\right]-\sum_{k=l}^{n} \phi\left[S_{(k)}=a^{\prime}\right]$ with $D_{n} \equiv D(1, n)$, and let $M_{l, n} \equiv \sum_{i=l}^{n} m_{i}$. By (R3) with $r=3$

$$
\begin{aligned}
E\left[D^{2}(l, n)\right]= & O\left(M_{l, n}\right) \\
& +2 \sum_{l \leq j<k \leq n}\left\{\left(P\left[S_{(k)}=a, S_{(j)}=a\right]-P\left[S_{(k)}=a^{\prime}, S_{(j)}=a\right]\right)\right. \\
& \left.\quad+\left(P\left[S_{(k)}=a^{\prime}, S_{(j)}=a^{\prime}\right]-P\left[S_{(k)}=a, S_{(j)}=a^{\prime}\right]\right)\right\} \\
= & O\left(M_{l, n}\right)+O\left[\sum _ { l \leq j < k \leq n } \left\{( k - j ) ^ { - 1 / 2 + \epsilon ^ { \prime } } \left(P\left[S_{(k)}=a, S_{(j)}=a\right]\right.\right.\right. \\
& \left.+P\left[S_{(k)}=a^{\prime}, S_{(j)}=a^{\prime}\right]\right) \\
& \left.\left.+(k-j)^{-3} P\left[S_{j} \in\left\{a, a^{\prime}\right\}\right]\right\}\right] \quad \forall \epsilon^{\prime}>0 \\
= & O\left(M_{l, n}\right)+O\left(M_{l, n}\right)+\sum_{j=l}^{n} m_{j}(a) \sum_{k=l}^{n} k^{-1 / 2+\epsilon^{\prime}} P\left[S_{(k)}=a \mid S_{(j)}=a\right] \\
& +\sum_{j=l}^{n} m_{j}\left(a^{\prime}\right) \sum_{k=l}^{n} k^{-1 / 2+\epsilon^{\prime} P\left[S_{(k)}=a^{\prime} \mid S_{(j)}=a^{\prime}\right] \quad \forall \epsilon^{\prime}>0 .}
\end{aligned}
$$

Applying $(\mathrm{H})$ and the hypothesis $(\dagger \dagger)$ of (2) to the last inequality gives

$$
\begin{aligned}
E\left[D^{2}(l, n)\right] & \leq K\left(M_{l, n}+M_{l, n}\left(M_{n}^{1+2 \epsilon}\right)^{1 / 2+\epsilon^{\prime \prime}}\right) \quad\left(\forall \epsilon^{\prime \prime}>0\right) \\
& \leq K\left(M_{l, n} M_{n}^{1 / 2+\epsilon+\epsilon^{\prime \prime}}\right) \quad\left(\forall \epsilon^{\prime \prime}>0\right) .
\end{aligned}
$$

An inequality that appears in Billingsley [2, p. 102] says that if $\left\{Y_{i}\right\}$ is a sequence of random variables for which $\exists$ a positive sequence $\left\{\mu_{i}\right\}$ and $\beta, \nu \geq 1$ :

$$
E\left(\left|\sum_{i=l}^{n} Y_{i}\right|^{\nu}\right) \leq\left(\sum_{i=l}^{n} \mu_{i}\right)^{\beta} \quad \forall l<n
$$

then 


$$
E\left(\max _{l \leq k \leq n} \sum_{i=l}^{k} Y_{i}\right)^{\nu} \leq \lg _{2}^{\nu}(4 n)\left(\sum_{i=l}^{n} \mu_{i}\right)^{\beta}
$$

If we take $\mu_{i} \equiv K m_{i} M_{n}^{1 / 2+\epsilon+\epsilon^{\prime}}, \nu=2$ and $\beta=1$ then $(*)$ shows that $(* *)$ holds for $Y_{i}=\phi\left[S_{i}=a\right]-\phi\left[S_{i}=a^{\prime}\right]$. Noting that the hypothesis on $X_{1}$ in (2) implies $n^{1-a}=O\left(M_{n}\right)$ we obtain

$$
\begin{aligned}
E\left(\max _{l \leq k \leq n} D_{k}\right)^{2} & =O\left(\lg _{2}^{2}(4 n) M_{n}^{3 / 2+\epsilon+\epsilon^{\prime \prime}}\right) \quad\left(\forall \epsilon^{\prime \prime}>0\right) \\
& =O\left(M_{n}^{3 / 2+\epsilon+\epsilon^{\prime \prime}}\right) \quad\left(\forall \epsilon^{\prime \prime}>0\right)
\end{aligned}
$$

Fix $\epsilon^{\prime \prime}$ and choose $n(k)$ so that $M_{n(k)} \sim k^{\lambda}$ where $\lambda>\left(\epsilon^{\prime \prime}\right)^{-1}$. Then $M_{n(k+1)} / M_{n(k)} \sim 1$ and so

$$
E\left(\max _{m(k) \leq n \leq n(k+1)} D_{n} / M_{n}^{(3 / 4)+(\epsilon / 2)+\epsilon^{\prime \prime}}\right)^{2}=O\left(M_{n(k)}^{-\epsilon^{\prime \prime}}\right)=O\left(k^{-\lambda \epsilon^{\prime \prime}}\right) .
$$

Since $\Sigma_{k} k^{-\lambda \epsilon "}<\infty$, the Markov inequality and the Borel-Cantelli lemma imply

$$
\max _{n(k) \leq n \leq n(k+1)}\left(D_{n} / M_{n}^{\left.(3 / 4)+(\epsilon / 2)+\epsilon^{\prime \prime}\right)} \underset{k \rightarrow \infty}{\longrightarrow} 0\right. \text { a.e. }
$$

Proof of (2) of Proposition 1. By (R2) we see

$$
\sum_{k=j+1}^{n} P\left[S_{(k)}=a \mid S_{(j)}=a^{\prime}\right] \leq \sum_{k=j+1}^{n} m_{k-j}(0)+2 s_{0}^{k-j}=O\left(M_{n}\right)
$$

which clearly means $(\dagger \dagger)$ holds with $\epsilon=0$.

Proof of (3) of Theorem 1. Let $\delta \in(0,1)$. For $k \in I^{+}$define $n(k)=$ $\max \left\{j: M_{j} \leq M_{k}^{\delta}\right\}$. Because $m_{j}<1$, we see $M_{n(k)} \sim M_{k}^{\delta}$. By hypothesis, ( $\left.\dagger\right)$ holds for $g(x)=\lg x$ and thus by (1) of Theorem 1

$$
\lim _{k}\left(\lg M_{k}\right)^{-1} \sum_{i=1}^{n(k)} \phi\left[S_{(i)}=a\right] / M_{i}=\delta
$$

Therefore

$$
\begin{aligned}
1-\delta & =\lim _{k}\left(\lg M_{k}\right)^{-1} \sum_{i=n(k)+1}^{k} \phi\left[S_{(i)}=a\right] / M_{i} \\
& \leq \frac{\lim }{k}\left(\sum_{i=1}^{n} \phi\left[S_{(i)}=a\right]\right) / M_{k}^{\delta} \lg M_{k},
\end{aligned}
$$


i.e.,

$$
P\left[\sum_{i=1}^{k} \phi\left[S_{(i)}=a\right]<M_{k}^{\delta^{\prime}} \text { i.o. }\right]=0 \text { for all } \delta^{\prime} \in(0,1) \text {. }
$$

This, combined with (2) of Theorem 1 yields (3) of Theorem 1.

Proof of Theorem 2. We will first prove (1) $\Leftrightarrow(2),(3) \Rightarrow(1)$ and (2) $\Rightarrow$ (3) establishing the equivalence of (1), (2) and (3) which do not involve statements about sample sequences of ruled sums. $(4 a) \Rightarrow(4 b),(5 a) \Rightarrow(5 b)$ and $(6 a) \Rightarrow(6 b)$ are obvious since the (b) statements are weakened forms of the (a) statements in which $\forall$ is replaced by $\exists$. We then show that $(4 b) \Rightarrow(2),(5 b) \Rightarrow(3),(6 b) \Rightarrow(2)$ follow from the monotone convergence theorem and the Borel-Cantelli iemma. Next we show that $(6 a) \Rightarrow(4 a)$ and $(5 a)$ by a Fubini's theorem argument. Finally we prove that $(1) \Rightarrow(6 a)$ which completes the demonstration of equivalence of the nine conditions of Theorem 2. The proof of $(1) \Rightarrow(6 a)$ relies on tools developed earlier, in particular Lemma 1 and Lemma 2.

(1) $\Leftrightarrow(2)$. By (C4),

$$
\sum_{n} \sum_{a} P^{2}\left[S_{n}=a\right]=(2 \pi)^{-1} \sum_{n} \int_{-\pi}^{\pi}|\hat{f}(\theta)|^{2 n} d \theta
$$

(3) $\Rightarrow(1)$. By (C2),

$$
\sum_{n} P\left[S_{n}=d_{n}\right] \leq(2 \pi)^{-1} \sum_{n} \int_{-\pi}^{\pi}|\hat{f}(\theta)|^{n} d \theta=(2 \pi)^{-1} \int_{-\pi}^{\pi}(1-|\hat{f}(\theta)|)^{-1} d \theta \text {. }
$$

(2) $\Rightarrow(3) . \forall n$ let $d_{n}: P\left[S_{n}=d_{n}\right]=\max _{a} P\left[S_{n}=a\right]$. Then

$$
\sum_{n} P\left[s_{n}=d_{n}\right] \geq \sum_{n} \sum_{a} P^{2}\left[s_{n}=a\right]=\infty .
$$

$(4 a) \Rightarrow(4 b),(5 a) \Rightarrow(5 b),(6 a) \Rightarrow(6 b)$. Obvious.

$(4 \mathrm{~b}) \Rightarrow(2)$. By the monotone convergence theorem

$$
\infty=E\left(\sum_{n} m_{n}\left(S_{(n)}\right)\right)=\sum_{n} \sum_{a} P^{2}\left[s_{n}=a\right] .
$$

$(5 b) \Rightarrow(3)$. By the Borel-Cantelli lemma

$$
P\left[S_{(n)}-d_{n}=a \text { i.o. }\right]>0 \Rightarrow \sum_{n} P\left[S_{(n)}=d_{n}+a\right]=\infty .
$$

$(6 \mathrm{~b}) \Rightarrow(2)$. By the Borel-Cantelli lemma

$$
P\left[S_{(n)_{1}}=S_{(n)_{2}}+a \text { i.o. }\right]>0 \Rightarrow \sum_{n} P\left[S_{(n)_{1}}=S_{(n)_{2}}+a\right]=\infty .
$$

Since the variables $S_{(n)_{1}}$ and $S_{(n)_{2}}$ are independent 


$$
\infty=\sum_{n} P\left[S_{(n)_{1}}=S_{(n)_{2}}+a\right]=\sum_{n} \sum_{b} m_{n}(a+b) m_{n}(b) \leq \sum_{n} \sum_{b} m_{n}^{2}(b)
$$

by $(a)$ of $(\mathrm{R} 0)$.

$(6 a) \Rightarrow(4 a)$ and $(5 a)$. Consider the maps $\sigma_{1}$ and $\sigma_{2}$ defined by $\sigma_{1}(n) \equiv 2 n+$ 1 and $\sigma_{2}(n) \equiv 2 n$ for all $n \in I^{+}$. Then by Theorem 6 in $\$$ III (which is independent of $\S$ II), we see the rules ()$_{1}$ and ()$_{2}$ gotten by $(n)_{i}=\bigcup_{j \in(n)} \sigma_{i}(j), i=1,2$, define two sequences of ruled sums equivalent to the sequence of ruled sums defined by ( ). These two rules, ()$_{1}$ and ()$_{2}$ have the property demanded in (6), i.e., $(n)_{1} \cap(m)_{2}=\varnothing$ for $n, m \in I^{+}$, and thus

$$
P\left[S_{(n)_{1}}=S_{(n)_{2}}+a, \text { i.o. }\right]=1 \text { for all } a \in I .
$$

We now use a standard Fubini argument often associated with symmetrization ([12, p. 245] or $\left[7\right.$, p. 7]). Consider the sample spaces $\Omega_{1}$ and $\Omega_{2}$ generated by $\bigcup_{i \in \cup_{n}(n)}\left\{X_{i}\right\}$ and $\bigcup_{i \in \cup_{n}(m)}\left\{X_{i}\right\}$ respectively. Then the total sample space $\Omega$ could be taken as $\Omega_{1} \times \Omega_{2}$ and the probability $P$ on $\Omega$ could be considered to be $P_{1} \times P_{2}$ where $P_{1}$ and $P_{2}$ are $P$ restricted to $\Omega_{1}$ and $\Omega_{2}$ respectively. For $a \in I$, we define

$$
A_{a} \equiv\left[\omega \in \Omega_{2}: P_{1}\left[S_{(n)_{1}}=S_{(n)_{2}}(\omega)+a \text { i.o. }\right]=1\right] .
$$

By $(*)$ and the Fubini theorem we see that $P\left[A_{a}\right]=P_{2}\left[A_{a}\right]=1$ for all $a \in I$.

For $a=0$, (4) follows for $\omega \in A_{a}$ by the easy half of the Borel-Cantelli lemma.

$I$ is a countable and so $P\left[\bigcap_{a} A_{a}\right]=1$. Letting $\omega \in \bigcap_{a} A_{a}$ and $d_{n} \equiv$ $S_{(n) 2}(\omega),(5)$ follows.

$(1) \Rightarrow(6 a)$. In Lemma 2 we let $Z_{j} \equiv S_{(j)_{2}}-S_{(j)_{1}}$, i.e., $Z_{j}$ is the sum of $j$ independent symmetrizations of $X_{1}$. Thus (b) of Lemma 2 is satisfied.

Letting $A \equiv(k)_{1}, B \equiv(k)_{2}, A^{\prime} \subseteq(k)_{1}-(k)_{1} \cap(j)_{1}, B^{\prime} \subseteq(k)_{2}-(k)_{2} \cap(j)_{2}$ with $\left|A^{\prime}\right|=\left|B^{\prime}\right|=k-j$ and letting $\mathbb{Q} \equiv\left[Z_{j}=a\right]$ in (R2b) we see

$$
P\left[Z_{k}=a, Z_{j}=a\right] \leq P\left[Z_{k-j}=0\right] P\left[Z_{j}=a\right]
$$

i.e., $P\left[Z_{k}=a \mid Z_{j}=a\right] \leq P\left[Z_{k-j}=0\right]$ which shows (a) of Lemma 2 is satisfied.

(1) implies $\int_{-\pi}^{\pi}\left(1-|\hat{f}(\theta)|^{2}\right)^{-1} d \theta=\infty$ and so by (C2), $M_{n} \vec{n} \infty$.

So letting $g(x)=\lg x$ in Lemmas 1 and 2 we see (1) implies (6a). In fact by (1) of Lemma 1 we have a "rate of occurrence" for these meetings $S_{(n)}=$ $S_{(n) 2}+a$.

Proof of Theorem 3. In Lemma 2, we let $Z_{n} \equiv\left(Y_{n}, V_{n}\right) \equiv\left(S_{(n)}-S_{(n)_{3}}\right.$, $\left.S_{(n)_{2}}-S_{(n)_{4}}\right)$. Thus $P\left[\left(S_{(n)_{1}}, S_{(n)_{2}}\right)=\left(S_{(n)_{3}}, S_{(n)_{4}}\right)\right.$ i.o. $]=P\left[Z_{n}=(0,0)\right.$ i.o. $]$. 
Using (R2b),

$$
\begin{aligned}
P\left[Z_{n}\right. & \left.=(0,0), Z_{j}=(0,0)\right]=P\left[Y_{n}=0, Y_{j}=0\right] P\left[V_{n}=0, V_{j}=0\right] \\
& \leq P\left[Y_{n-j}=0\right] P\left[Y_{j}=0\right] P\left[V_{n-j}=0\right] P\left[V_{j}=0\right] \\
& =P\left[Z_{j}=(0,0)\right] P\left[Z_{n-j}=(0,0)\right] .
\end{aligned}
$$

Thus (a) of Lemma 2 is satisfied and so for $g(x)=\lg x$, Lemma 1 and Lemma 2 show that $P\left[Z_{n}=(0,0)\right.$ i.o. $]=1$ iff $\sum_{n} P\left[Z_{n}=(0,0)\right]=\infty$. However, by (C4),

$$
\begin{aligned}
\sum_{n} P\left[Z_{n}=\right. & (0,0)]=\sum_{n}\left(\sum_{a} P^{2}\left[S_{n}=a\right]\right)^{2} \\
= & (2 \pi)^{-2} \sum_{n} \int_{-\pi}^{\pi} \int|\hat{f}(\theta)|^{2 n}|\hat{f}(\phi)|^{2 n} d \theta d \phi=\infty \\
& \text { iff } \int_{-\pi}^{\pi} \int(1-|\hat{f}(\theta)||\hat{f}(\phi)|)^{-1} d \theta d \phi=\infty .
\end{aligned}
$$

We have proved (1), (2) and (3) of Theorem 3 are equivalent.

We now show (4) is equivalent to (1) and (2). First, by the Schwarz inequality,

$$
\sum_{n}\left(\sum_{a} m_{n}^{2}(a)\right)^{2} \leq \sum_{n}\left(\sum_{a} m_{n}^{3}(a)\right)\left(\sum_{a} m_{n}(a)\right)=\sum_{a} \sum_{n} m_{n}^{3}(a) .
$$

On the other hand, since the Fourier transform of a product of two functions is the convolution of their Fourier transforms,

$$
\sum_{a} m_{n}^{3}(a)=(2 \pi)^{-2} \iint \hat{f}^{n}(\theta) \overline{\hat{f}^{n}}(\theta-\phi) \hat{\hat{f}}^{n}(\phi) d \theta d \phi
$$

by Plancherel's theorem (C4). Since $\left|\overline{\hat{f}^{n}}(\theta) \overline{\hat{f}^{n}}(\theta-\phi) \overline{f^{n}}(\phi)\right| \leq\left|\hat{f}^{n}(\theta) \hat{f}^{n}(\phi)\right|$,

$$
\begin{aligned}
& \left|\sum_{n} \iint \overline{\hat{f}}^{n}(\theta) \overline{\hat{f}^{n}}(\theta-\phi) \overline{\hat{f}}^{n}(\phi) d \theta d \phi\right| \\
& \quad \leq \sum_{n} \iint\left|\hat{f}^{n}(\theta)\right|\left|\hat{f}^{n}(\phi)\right| d \theta d \phi=\iint(1-|\hat{f}(\theta)||\hat{f}(\phi)|)^{-1} d \theta d \phi .
\end{aligned}
$$

Hence

$$
\sum_{n} \sum_{a} m_{n}^{3}(a), \quad \sum_{n}\left(\sum_{a} m_{n}^{2}(a)\right)^{2} \text { and } \iint(1-|\hat{f}(\theta) \hat{f}(\phi)|)^{-1} d \theta d \phi
$$

are infinite together completing the proof that (1)-(4) are equivalent.

We now show (5) is equivalent to (4) to complete the proof of Theorem 3. In

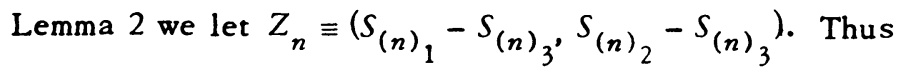




$$
\begin{aligned}
& P\left[\left(S_{(n)_{1}}=S_{(n)_{2}}=S_{(n)_{3}} \text { i.o. }\right]\right. \\
& \quad=P\left[\left(S_{(n)_{1}}-S_{(n)_{3}}=0, S_{(n)_{2}}-S_{(n)_{3}}=0\right) \text { i.o. }\right]=P\left[Z_{n}=(0,0) \text { i.o. }\right] .
\end{aligned}
$$

Let $X, X^{\prime}, X^{\prime \prime}$ be independent and distributed like $X_{1}$. To show (a) of Lemma 2 is satisfied, we take, in (R2c), $\mathbb{C}=\left[Z_{j}=(0,0)\right], A=(n)_{1}, B=(n)_{2}, C=(n)_{3}$, $A^{\prime} \subseteq(n)_{1}-(j)_{1}, B^{\prime} \subseteq(n)_{2}-(j)_{2}, C^{\prime} \subseteq(n)_{3}-(j)_{3}$ with $\left|A^{\prime}\right|=\left|B^{\prime}\right|=\left|C^{\prime}\right|=n-j$. By the hypothesis of Theorem $3, A, B$ and $C$ are pairwise disjoint and $A^{\prime}, B^{\prime}$ and $C^{\prime}$ are independent of $\mathbb{Q}$. By $(\mathrm{R} 2 \mathrm{c})$, the definition of $Z_{n}$ and the first sentence of this paragraph

$$
P\left[Z_{j}=(0,0), Z_{n}=(0,0)\right] \leq P\left[Z_{j}=(0,0)\right] P\left[Z_{n-j}=(0,0)\right]
$$

i.e., (a) of Lemma 2 is satisfied. For $g(x)=\lg x$, Lemma 1 and Lemma 2 now show that $P\left[Z_{n}=(0,0)\right.$ i.o. $]=1$ iff $\Sigma_{n} P\left[Z_{n}=(0,0)\right]=\infty$, i.e., iff $\Sigma_{n} \Sigma_{a} m_{n}^{3}(a)=\infty$ by definition of $Z_{n}$. This completes the proof of the equivalence of (1)-(5) of Theorem 3. (1) of Lemma 1 actually establishes a "rate of occurrence" for these triple meetings $S_{(n)_{1}}=S_{(n)_{2}}=S_{(n)_{3}}$.

The conditions in Lemma 2 yield a rate of visitation in Theorem 1 . For Theorem 4, the variables are not necessarily lattice and we are only interested in infinite visitation so we use a somewhat different method based on Lemma 3.

Lemma 3. Let $\left\{x_{n}\right\}$ be a sequence of independent random variables, let $\left\{B_{n}\right\}$ be a sequence of open intervals in $R$, and let $\left\{f_{n}\right\}$ be a sequence of functions $X_{i=1}^{\infty} R_{i} \rightarrow R$. Let $Z_{n} \equiv f_{n}\left(X_{1}, \ldots, X_{n}, \ldots\right)$ and for $x_{1}, \ldots, x_{m} \in R$ let $Y_{n} \equiv$ $Y_{n}\left(x_{1}, \ldots, x_{m}\right) \equiv f_{n}\left(x_{1}, \ldots, x_{m}, X_{m+1}, \cdots\right)$. If $\exists A>0: \forall m \in I^{+}, x_{1}, \cdots, x_{m}$ $\in R$

(a) $\exists \Gamma>0$ and $n_{0}: \forall n>n_{0} P\left[Y_{n} \in B_{n}\right] \geq \Gamma P\left[Z_{n} \in B_{n}\right]$,

(b) $\exists$ a subsequence $n(k)$ of $I^{+}$:

$$
\max _{1 \leq j<n(k)} \sum_{l=j+1}^{n(k)} P\left[Y_{l} \in B_{l} \mid Y_{j} \in B_{j}\right] \leq A \sum_{l=1}^{n(k)} P\left[Y_{l} \in B_{l}\right]
$$

then

$$
P\left[Z_{j} \in B_{j} \text { i.o. }\right]={ }_{0}^{1} \text { iff } \sum P\left[Z_{j} \in B_{j}\right]_{<\infty}^{=\infty} .
$$

Proof. Since $\Sigma P\left[Z_{j} \in B_{j}\right]<\infty \Rightarrow P\left[Z_{j} \in B_{j}=0\right.$ i.o. $]=0$ by the Borel-Cantelli lemma we assume $\Sigma P\left[Z_{j} \in B_{j}\right]=\infty$. By (a) this implies $\Sigma P\left[Y_{j} \in B_{j}\right]=\infty$. Hence 
(*) $P\left[\overline{\lim }\left(\sum_{j=1}^{n} \phi\left[Y_{j} \in B_{j}\right] / \sum_{j=1}^{n} P\left[Y_{j} \in B_{j}\right]\right)>0\right]$

$$
\leq P\left[Y_{j} \in B_{j} \text { i.o. }\right]=P\left[Z_{j} \in B_{j} \text { i.o. } \mid X_{1}=x_{1}, \ldots, X_{m}=x_{m}\right]
$$

since the $X_{i}$ are independent.

Kochen and Stone [9] showed that for any sequence of random variables $W_{j}$

$$
\begin{aligned}
P[\varlimsup & \left.\lim \sum_{j=1}^{n} \phi\left[W_{j} \in B_{j}\right] / \sum_{j=1}^{n} P\left[W_{j} \in B_{j}\right]>0\right] \\
& \geq \overline{\lim }\left(\sum_{j=1}^{n} P\left[W_{j} \in B_{j}\right]\right)^{2} / \sum_{i=1, j=1}^{n} P\left[W_{i} \in B_{i}, W_{j} \in B_{j}\right] .
\end{aligned}
$$

Taking $W_{j} \equiv Y_{j}$ and letting $N_{n}=\sum_{j=1}^{n} P\left[Y_{j} \in B_{j}\right],(*)$ says

$$
P\left[\left[Z_{j} \in B_{j} \text { i.o. }\right] \mid X_{1}=x_{1}, \ldots, X_{m}=x_{m}\right] \geq \lim _{n} N_{n}^{2} / \sum_{i, j=1}^{n} P\left[Y_{i} \in B_{i}, Y_{j} \in B_{j}\right]
$$

while (b) gives

$$
\begin{aligned}
& \sum_{i, j=1}^{n(k)} P\left[Y_{i} \in B_{i}, Y_{j} \in B_{j}\right] \\
& \quad=o\left(N_{n(k)}^{2}\right)+2 \sum_{j=1}^{n(k)} P\left[Y_{j} \in B_{j}\right] \sum_{i=j+1}^{n(k)} P\left[Y_{i} \in B_{i} \mid Y_{j} \in B_{j}\right] \leq o\left(N_{n(k)}^{2}\right)+2 A N_{n(k)}^{2} .
\end{aligned}
$$

Thus $\forall m \in I^{+}, \forall x_{1}, \ldots, x_{m} \in R, P\left[\left[Z_{j} \in B_{j}\right.\right.$ i.o. $\left.] \mid X_{1}=x_{1}, \ldots, X_{m}=x_{m}\right] \geq$ $(1 / 2 A)$. By a corollary of the martingale convergence theorem $[3, \mathrm{p} .93]$,

$$
P\left[\left[Z_{j} \in B_{j} \text { i.o. }\right] \mid X_{1}, \ldots, X_{m}\right] \underset{m}{\rightarrow} \phi\left(Z_{i} \in B_{j} \text { i.o. }\right)
$$

a.e. Now $\phi\left[Z_{j} \in B_{j}\right.$ i.o. $]$ is an indicator function and thus takes only the values 0 or 1 . However, we have shown $\phi$ to be $\geq(1 / 2 A)$ a.e. and thus $\phi$ equals 1 a.e. The proof is complete.

Proof of Theorem 4. To prove this theorem, we first note the following:

Note 1. Since $a_{n}=o(n)$, we see that for $0<\epsilon<|c|, \exists n_{0}>0$ such that $n>n_{0} \Rightarrow\left[\left|S_{(n)} / a_{n}-d\right|<\epsilon\right] \subseteq\left[\left|\left(S_{(n)}-n c\right) / n\right|>\epsilon\right]$.

Note 2. A theorem of Katz [11] states: For $\alpha \geq 1$,

$$
E X^{a}<\infty \text { iff } \sum_{k=1}^{\infty} k^{a-2} P\left[\left|\frac{S_{k}-k E X_{1}}{k}\right|>\epsilon\right]<\infty .
$$


Note 3. This note was pointed out to us by K. G. Binmore and M. Katz. A result of Stone [19] applied to nonlattice variables $X_{i}$ with $E\left(X_{i}\right)=0, E\left(X_{i}^{2}\right)=1$ states $P\left[\sqrt{n} x \leq S_{n} \leq \sqrt{n}(x+b)\right]=(1 / \sqrt{2 \pi}) \int_{x}^{x+b} e^{-x^{2} / 2}+o_{n}(1)(b+1 / \sqrt{n})$ where o(1) is uniform in $x, b$. Define $x, b$ by $\sqrt{n} x=b_{n}, \sqrt{n}(x+b)=c_{n}$. Then if $b(n), c(n)=$ $o\left(n^{1 / 2}\right)$ and $c(n)-b(n) \geq K>0$ the Stone theorem yields $P\left[b(n)<S_{n}<c(n)\right] \sim$ $(c(n)-b(n))(2 \pi n)^{-1 / 2}$. (A similar result holds for lattice random variables (Gnedenko and Kolmogoroff $[10$, p. 233]).)

(4) $\Rightarrow(1)$ and (2) $\Rightarrow(1)$. In Note 1 , let $c=E X_{1}$ and $d=0$ if (4) holds, $d=b$ if (2) holds and then combine this with Note 2 and $a=2$ to get a contradiction.

(3) $\Rightarrow(2)$ and (3) $\Rightarrow(4)$ are easy. To complete the proof of equivalence we show $(1) \Rightarrow(3)$.

$(1) \Rightarrow$ (3). In Lemma 3 , let $f_{n}\left(x_{1}, \ldots, x_{l}, \ldots\right) \equiv a_{n}^{-1} \Sigma_{i \epsilon(n)} x_{i}$. Further let $m \in I^{+} ; x_{1}, \cdots, x_{m} \in R ; Y_{n} \equiv Y_{n}\left(x_{1}, \cdots, x_{m}\right)$, and $B_{n} \equiv(b-\epsilon, b+\epsilon)$ for all $n$. Let $A_{n}(b, \epsilon) \equiv\left[\left|Y_{n}\left(x_{1}, \cdots, x_{m}\right)-b\right|<\epsilon\right] \equiv\left[Y_{n} \in B_{n}\right]$.

Now in Note 3 , let $b(n) \equiv a_{n}(b-\epsilon)-\Sigma_{i \epsilon(n) \cap m} x_{i}$ and $c(n) \equiv a_{n}(b+\epsilon)-$ $\Sigma_{i \in(n) \cap^{\prime} m} x_{i}$. Since $m$ is fixed, we see

$$
P\left[Y_{n} \in B_{n}\right]=P\left[b(n)<S_{|(n)-m n(n)|}<c(n)\right] \sim 2 \epsilon a_{n}(2 \pi n)^{-1 / 2}
$$

(i.e., it is asymptotically independent of $x_{1}, \cdots, x_{m}$ and b). Hence since $a_{n}$ is nondecreasing we have

$$
\sum_{k=j+1}^{n} P\left[A_{k}(b, \epsilon) \mid A_{j}(b, \epsilon)\right] \leq \sum_{k=j+1}^{n} P\left[\left|S_{k-j}-b\left(a_{k}-a_{j}\right)\right| \leq\left(a_{k}+a_{j}\right) \epsilon\right]
$$

$$
=O\left(\sum_{k=j+1}^{n} a_{k}(k-j)^{-1 / 2}\right)=O\left(a_{n} n^{1 / 2}\right)
$$

(**) $\quad \sum_{k=1}^{n} P\left[A_{k}(b, \epsilon)\right] \geq \sum_{k=n / 2}^{n} P\left[A_{k}(b, \epsilon)\right] \geq K \sum_{k=n / 2}^{n} a_{k} k^{-1 / 2} \geq K a_{n / 2} n^{1 / 2}$.

Thus to finish the proof of Theorem 4 , we combine $(*)$ and $(* *)$ with Lemma 3 where $n(k)$ is a subsequence of $I^{+}$with the property that $a_{n(k)}<C a_{n(k) / 2}$ for some fixed positive constant $C$. Such a sequence $n(k)$ exists since even $\underline{\lim } a_{2 k} / a_{k}>\sqrt{2} \Rightarrow \underline{\lim } a_{2^{n}} /\left(2^{n}\right)^{1 / 2}=\infty$ and this contradicts $a_{n}=o\left(n^{1 / 2}\right)$.

Proof of Theorem 5. If $\hat{f}(2 \pi t) \neq 1$,

$$
\begin{aligned}
E\left|\frac{1}{N} \sum_{n=1}^{N} e^{2 \pi i t S}(n)\right|^{2} & =\frac{1}{N^{2}} \sum_{m=1}^{N} \sum_{n=1}^{N} E\left(e^{2 \pi i t(S(n)-S(m)}\right) \\
& \leq \frac{1}{N^{2}} \sum_{m=1}^{N} \sum_{n=1}^{N}|f|^{|n-m|}(2 \pi t) \leq \frac{N}{N^{2}} \frac{2}{1-|f|(2 \pi t)}=O\left(\frac{1}{N}\right) .
\end{aligned}
$$


We conclude $(1 / N) \sum_{n=1}^{N} e^{2 \pi i t S}(n) \rightarrow 0$ a.e., by Lemma 0 with $N_{k}=k^{2}$, since

$$
\begin{aligned}
& \max _{N=N_{k}}^{N_{k+1}}\left|\frac{1}{N} \sum_{n=1}^{N} e^{2 \pi i t S(n)}-\frac{1}{N_{k}} \sum_{n=1}^{N_{k}} e^{2 \pi i t S}(n)\right| \\
& \quad \max _{N=N_{k}}^{N+1}\left|\frac{1}{N_{k}}\left(\sum_{n=1}^{N}-\sum_{n=1}^{N} e^{2 \pi i t S(n)}\right)+\left(\frac{1}{N}-\frac{1}{N_{k}}\right) \sum_{n=1}^{N} e^{2 \pi i t S(n)}\right| \\
& \leq(2 k+1) / k^{2}+2 / k=o(1) .
\end{aligned}
$$

By the hypothesis, a.e., $\forall l \in l, N^{-1} \sum_{n=1}^{N} e^{2 \pi i(l / a) S}(n) \rightarrow 0$. This is equivalent to $S_{(n)}$ equidistributed $\bmod (0, a)$ a.e. [20].

III. Equivalence relations and partial orderings on rules. In this section we consider the question of when two rules may be considered equivalent from the viewpoint of ruled sums. In Theorem 6 we show that two natural definitions lead to the same equivalence relation. Theorem 6 is stated for lattice random variables for convenience. We then consider a weaker equivalence relation and associated partial ordering for Gaussian variables and prove in Theorem 7 an extension of a theorem of [1].

The orem 6. Let ( ) and ( )' be two rules. Then conditions (a) and (b) are equivalent. $(n)\}$,

(a) $\exists$ a 1-1 map $\sigma$ from $\bigcup_{j=1}^{\infty}(j)$ onto $\bigcup_{i=1}^{\infty}(i)^{\prime}: \forall n \in I^{+}(n)^{\prime}=\{\sigma(i), i \epsilon$

(b) $\forall j_{,} i_{1}, \ldots, i_{j} \in I^{+}\left|\left(i_{1}\right) \cap \ldots \cap\left(i_{j}\right)\right|=\left|\left(i_{1}\right)^{\prime} \cap \ldots \cap\left(i_{j}\right)^{\prime}\right|$.

$S_{(n)}, n=1,2, \cdots$, and $S_{(n)}, n=1,2, \cdots$, define equivalent stochastic processes for all distributions $m(a)=P\left(X_{1}=a\right)$ iff one and bence both of (a) and (b) bold.

Proof. That $(a) \Rightarrow\left(\right.$ b) is clear since $\sigma\left\{\left(i_{1}\right) \cap \ldots \cap\left(i_{j}\right)\right\}=\sigma\left(\left(i_{1}\right)\right) \cap \ldots \cap \sigma\left(\left(i_{j}\right)\right)$ $=\left(i_{1}\right)^{\prime} \cap \ldots \cap\left(i_{j}\right)^{\prime}$.

To prove the converse, we first show that a mapping can always be defined if we restrict attention to any finite number $n$ of sets $\left(i_{1}\right), \ldots,\left(i_{n}\right)$.

Lemma 4. If $V_{1}, \cdots, V_{n}$ are $n$ finite sets, then the $2^{n}$ cardinalities $\left|V_{i_{1}} \cap \ldots \cap V_{i_{j}} \cap \bigcup_{i=1}^{n} V_{i}\right|, j=0, \cdots, n$, and the $2^{n}$ cardinalities $\mid V_{1}^{\delta} \cap \cap V_{2}^{\delta_{2}} \cap$ $\cdots \cap V_{n}^{\delta_{n}} \cap \bigcup_{n=1}^{n} V_{i} \mid$ where each $\delta_{j}$ varies over the two values complementation $(=0)$ or its absence $(=1)$ determine each otber uniquely.

Proof. We denote $\left|V_{i_{1}} \cap \cdots \cap V_{i_{j}}\right|$ by $R_{u}$ where $u=\vec{u}$ is an $n$-long binary index $\left(u_{1} \cdots u_{n}\right)$ with $u_{k}=1$ for $k=i_{1}, \cdots, i_{j}$ and $u_{k}=0$ otherwise. We 
denote $\left|V_{1}^{\delta_{1}} \cap \ldots \cap V_{n}^{\delta} \cap \bigcup_{i=1}^{n} V_{i}\right|$ by $D_{\delta}$ where $\delta=\left(\delta_{1}, \ldots, \delta_{n}\right)$.

The $2^{n}$ vector $R$ is clearly obtained from the $2^{n}$ vector $D$ by a linear transformation $A, R=A D$, viz. $R_{u}=\Sigma_{u \leq \delta} D_{\delta}$, where $u \leq \delta$ is defined as $u_{k} \leq$ $\delta_{k}$ for all $k=1, \cdots, n$. Thus $D$ determines $R$ uniquely. Conversely we show $\operatorname{det} A=1$ so that $D=A^{-1} R$ and $R$ determines $D$ uniquely. For $n=1 \operatorname{det} A=1$. If the result is true for $n$ and $A_{n}$ is the $2^{n} \times 2^{n}$ matrix effecting the transformation from $D$ to $R$, for $n+1$ the $2^{n+1} \times 2^{n+1}$ matrix is

$$
\left(\begin{array}{cc}
A_{n} & A_{n} \\
0 & A_{n}
\end{array}\right)
$$

whose determinant $=\left(\operatorname{det} A_{n}\right)^{2}=1$ completing the induction.

We now complete the proof that $(\mathrm{b}) \Rightarrow(\mathrm{a})$ in Theorem 6 . For any $m \in I^{+}$ the $2^{m}$ sets $(1)^{\delta} 1 \cap \ldots \cap(m)^{\delta_{m}}, \delta_{i}=0,1, i=1, \cdots, m$, are disjoint so by Lemma 4 we may define a $1-1$ map $\tau$ from $\bigcup_{k=1}^{m}(k)$ onto $\bigcup_{k=1}^{m}(k)^{\prime}$ preserving cardinalities of intersections of sets $(i), i=1, \cdots, m$, by mapping the elements in (1) ${ }^{\delta} 1 \cap \ldots \cap(m){ }_{m}, 1-1$ onto the elements in (1) ${ }^{\prime} \delta_{1} \cap \ldots \cap(m){ }^{\prime} \delta_{m}$ for $\delta_{1}, \ldots, \delta_{m}=0,1$. In the set of all such mappings $\tau=\tau_{m}$ for any $m \in I^{+}$we define $\tau_{m}<\tau_{n}^{*}$ if $m<n$ and $\tau_{n}^{*}=\tau_{m}$ on domain $\tau_{m}$. For each $m=1,2, \ldots$ there are only finitely many mappings $\tau_{m}$ and there exist mappings $\tau_{n}$ for arbitrarily large $n$. Hence we can choose an infinite chain $\tau_{1}<\tau_{2}<\ldots$. For $i \in \bigcup_{k=1}^{\infty}(k)$ we may define $\tau(i)=\tau_{k}(i)$ where $i \in \operatorname{dom} \tau_{k^{\prime}} \cdot \tau$ necessarily maps $\bigcup_{k=1}^{\infty}(k) 1-1$ onto $\bigcup_{k=1}^{\infty}(k)^{\prime}$. Thus $(\mathrm{b}) \Rightarrow(\mathrm{a})$.

If (a) and (b) hold for ( ) and ( )', then for $P\left(X_{1}=a\right)=m(a)$ for any $j, n_{1}$ $\cdots n_{j} \in I^{+}$and $a_{1} \cdots a_{j} \in I$ we have

$$
\begin{aligned}
P\left[S_{\left(n_{1}\right)}\right. & \left.=a_{1}, \ldots, S_{\left(n_{j}\right)}=a_{j}\right]=P\left[S_{\left(n_{1}\right)^{\prime}}=a_{1}, \ldots, S_{\left(n_{j}\right)^{\prime}}=a_{j}\right] \\
& =\sum_{\left(\Sigma_{\delta \Sigma_{i}}{ }^{x} \delta=a_{i}\right)_{i=1,2, \cdots, j}} \prod_{\delta} m_{|\delta|}\left(x_{\delta}\right)
\end{aligned}
$$

where $\delta=\left\{\delta_{k}, k=1, \ldots, j\right\},|\delta|=\left|\bigcap\left(n_{k}\right)^{\delta}{ }_{k} \cap \bigcup_{k=1}^{j}\left(n_{k}\right)\right|$ and $v_{i}$ is a $j$-vector with 1 in the $i$ th place and 0 's elsewhere. Since all finite-dimensional probabilities are equal, $S_{(n)}$ and $S_{(n)}$, define equivalent processes. Conversely if (a) and (b) fail to hold it is clear we can find a distribution $m(a)$ and $n_{1} \cdots n_{j}$ : $P\left[S_{(n)_{1}}=a_{1}, \ldots, S_{\left(n_{j}\right)}=a_{j}\right] \neq P\left[S_{\left(n_{1}\right)},=a_{1}, \ldots, S_{\left(n_{j}\right)},=a_{j}\right]$. This completes the proof of Theorem 6 .

If either and hence both of (a) and (b) hold, we say ()$\cong()^{\prime}$. A weaker equivalence relation $\simeq$ defined by ()$\simeq()^{\prime}$ iff $\forall i, j \in I^{+}|(i) \cap(j)|=\mid(i)^{\prime} \cap$ $(j) '$ is sufficient when the underlying variable $X_{1}$ is Gaussian since the $S_{(j)}$ 
are jointly Gaussian and the covariances $E\left(S_{(i)} S_{(j)}\right)=|(i) \cap(j)|$ define the process.

An example of two non $\cong$ rules which are $\simeq$ is easy. Let $(2)=\{2,3\},(3)=$ $\{3,4,5\},(4)=\{2,4,6,7\},(2)^{\prime}=\{2,3\},(3)^{\prime}=\{3,4,5\},(4)^{\prime}=\{3,6,7,8\}$ with $(n)=(n)^{\prime}$ for $n \neq 2,3,4$ involving integers not appearing above. The pairwise intersections above have cardinality 1 , but $|(2) \cap(3) \cap(4)|=0 \neq 1=\mid(2)^{\prime} \cap(3)^{\prime}$ $\cap(4) \%$.

Corresponding respectively to the two equivalence relations $\cong$ and $\simeq$ on rules there are two natural partial orderings

$$
() \leqq()^{\prime} \text { iff } \forall j, i_{1}, \cdots, i_{j} \in I^{+} \quad\left|\left(i_{1}\right) \cap \cdots \cap\left(i_{j}\right)\right| \leq\left|\left(i_{1}\right)^{\prime} \cap \cdots \cap\left(i_{j}\right)^{\prime}\right|
$$

convenient for general distribution $m(a)$ and

$$
() \leq()^{\prime} \text { iff } \forall i, j \in I^{+} \quad|(i) \cap(j)| \leq\left|(i)^{\prime} \cap(j)^{\prime}\right| \text {. }
$$

We have at present no good theorem on $\leqq$ although natural conjectures abound and so results should be forthcoming. The following results concern $\leq$.

Proof of Proposition (2) stated in introduction. If the $2 \times 2$ covariance matrix for $S_{(j)}$ and $S_{(n)}$ is $\leq$ the matrix for $S_{(j)}$ ' and $S_{(n)}$ ', then following Slepian's method [18]

$$
\begin{aligned}
(d / d \lambda) & P_{\lambda}\left[S_{(j)} \in(u, v), S_{(n)} \in(u, v)\right] \\
& =\left(g_{\lambda}(u, u)+g_{\lambda}(v, v)-g_{\lambda}(u, v)-g_{\lambda}(v, u)\right) \cdot\left(E S_{(n)^{\prime}} S_{(j)^{\prime}}-E S_{(n)} S_{(j)}\right)
\end{aligned}
$$

(where $P_{\lambda}$ and $g_{\lambda}$ denote respectively probabilities and densities computed according to a covariance matrix with off diagonal entry $=(1-\lambda) E\left(S_{(j)} S_{(n)}\right)+$ $\left.\lambda E\left(S_{(n)}, S_{(j)}\right)\right)$. The proposition now follows from Lemma 5.

Lemma 5. Let $0 \leq c \leq \min (a, b)$. Then for all real $u, v, 0 \leq e^{-(a+b-2 c) u^{2}}+$ $e^{-(a+b-2 c) v^{2}}-e^{-\left(a u^{2}+b v^{2}-2 c u v\right)}-e^{-\left(a v^{2}+b u^{2}-2 c u v\right)}$.

Proof.

$$
\begin{aligned}
0 & \leq\left[e^{-(a-c) u^{2}}-e^{-(a-c) v^{2}}\right]\left[e^{-(b-c) u^{2}}-e^{-(b-c) v^{2}}\right] \\
& =e^{-(a+b-2 c) u^{2}}+e^{-(a+b-2 c) v^{2}}-e^{-\left((a-c) u^{2}+(b-c) c^{2}\right)}-e^{-\left((a-c) v^{2}+(b-c) u^{2}\right)},
\end{aligned}
$$

because the two factors on the right of the inequality are both positive or both negative since $a-c \geq 0, b-c \geq 0$. Hence it is sufficient to show that

$$
\begin{aligned}
e^{-\left((a-c) u^{2}+(b-c) v^{2}\right)} & +e^{-\left((a-c) v^{2}+(b-c) u^{2}\right)} \\
& \geq e^{-\left(a u^{2}+b v^{2}-2 c u v\right)}+e^{-\left(a v^{2}+b u^{2}-2 c u v\right)} .
\end{aligned}
$$

However, the first term on the left is $\geq$ the first term on the right because 


$$
\left((a-c) u^{2}+b-c\right) v^{2}-\left(a u^{2}+b v^{2}-2 c u v\right)=-c\left(u^{2}+v^{2}-2 u v\right)=-c(u-v)^{2} \leq 0
$$

since $c \geq 0$ and the same relationship holds between the second terms on the left and right completing the proof.

An extension of Proposition 2 to the joint distribution of $S_{(n)}$ 's taken 3 at a time is false. In fact, let $S_{(1)}=X_{1}, S_{(2)}=X_{2}+X_{3}, S_{(3)}=X_{1}+X_{2}+X_{3}$. Then for small positive $\epsilon$

$$
\begin{aligned}
P\left[\left|S_{(1)}\right|\right. & \left.<\epsilon,\left|S_{(2)}\right|<\epsilon,\left|S_{(3)}\right|<\epsilon\right] \\
& \geq P\left[0 \leq X_{1}<\epsilon / 2\right] P\left[0 \leq X_{2}+X_{3}<\epsilon / 2\right] \sim(1 / 2 \pi)(\epsilon / 2)^{2}(1 / \sqrt{2})
\end{aligned}
$$

while

$$
\begin{aligned}
& P\left[\left|S_{1}\right|<\epsilon,\left|S_{2}\right|<\epsilon,\left|S_{3}\right|<\epsilon\right] \\
& \quad \leq P\left[\left|X_{1}\right|<\epsilon\right] P\left[\left|X_{2}\right|<2 \epsilon\right] P\left[\left|X_{3}\right|<2 \epsilon\right] \sim(1 \sqrt{2 \pi})^{3}(2 \epsilon)(4 \epsilon)(4 \epsilon) \\
& \quad=\left(32 / \sqrt{2 \pi}^{3}\right) \epsilon^{3}<(\epsilon / 2)^{2} /(2 \pi \sqrt{2}) \text { for } \epsilon \text { sufficiently small. }
\end{aligned}
$$

In fact this same argument gives the same inequality for general $X_{1}$ with continuous distribution and the above rules.

Proof of Theorem 7 stated in the introduction. By hypothesis the $(N-n+1)$ $\times(N-n+1)$ covariance matrix of $S_{(n)}, \ldots, S_{(N)}$, dominates the covariance matrix of $S_{(n)}, \cdots, S_{(N)}$. Hence by Slepian's theorem [18, p. 468] for any $c_{n^{\prime}}$ $\cdots, c_{N}, P\left[s_{(n)} \leq c_{n}, \ldots, s_{(N)}, \leq c_{N}\right] \geq P\left[s_{(n)} \leq c_{n}, \ldots, S_{(N)} \leq c_{N}\right]$. Thus, choosing $c_{n}=a_{n} \lambda$,

$$
P\left[\overline{\lim } S_{(n)^{1}} / a_{n} \leq \lambda\right] \geq P\left[\overline{\lim } S_{(n)} / a_{n} \leq \lambda\right] .
$$

Since $\operatorname{Tim} S_{(n)} / a_{n}$ is a constant by the Kolmogoroff 0.1 law, Theorem 7 is proved.

IV. Tail $\sigma$-fields for ruled sums. Let $\mathfrak{T}()$ denote the tail $\sigma$-field for $S_{(n)}$; i.e., $\mathfrak{T}()=\bigcap_{n} \mathbb{B}\left(S_{(n)}, S_{(n+1)}, \ldots\right)$ where $\mathbb{B}\left(S_{(n)}, S_{(n+1)}, \ldots\right)$ is the Borel $\sigma$-field generated by $S_{(n)}, S_{(n+1)}, \ldots$ For both $S_{(n)}$ and $S_{n}$ this tail $\sigma$-field is trivial by the Hewitt-Savage theorem. However, this is not the case for other rules, even rules closely related to $\langle n\rangle$.

Let $Y$ be a variable independent of the $X_{i}$, all variables having the same distribution and $E X_{1}=0, E X_{1}^{2}=1$ and define $S_{(n)}=Y+S_{\langle n-1\rangle}, n=1,2, \ldots$, so that $S_{(n)}$ differs from the rule $S_{\langle n\rangle}$ only in the presence of a single permanent variable $Y$. We show that $Y \in \mathscr{T}()$ by showing that $Y \in \mathbb{B}\left(S_{(n)}, S_{(n+1)}, \ldots\right)$ for all $n$. In fact, consider

$$
Z_{N}=\sum_{j=n}^{N} \frac{1}{j-1} S_{(j)} \in \mathbb{B}\left(S_{(n)}, S_{(n+1)}, \ldots\right) .
$$


Now $E\left[\left(Z_{n} / \log N-Y\right)^{2}\right]=o(1)$, and so by Chebyshev's inequality, $Z_{N} / \log N \stackrel{P}{\rightarrow} Y$. But then a subsequence converges a.s. to $Y$ which establishes that $Y \in \mathfrak{F}$.

Since for $m$ fixed $E\left(S_{(n)} S_{(m)} / \sqrt{n m}\right)=(1 / \sqrt{n m}) \rightarrow 0$ as $n \rightarrow \infty$, this same example shows that $\forall m \operatorname{cov}\left(S_{(m)} S_{(n)}\right) \quad \vec{n} 0$ is not sufficient to insure that $\mathfrak{T}()$ is trivial even if the $X_{i}$ 's were $\Re(0,1)$. However, for normal variables $X_{i}$, $\mathfrak{T}$ will be trivial if $\forall m \max _{Y_{m}}, Z_{n} \operatorname{cov}\left(Y_{m}, Z_{n}\right) \rightarrow 0$ as $n \rightarrow \infty$, where $Y_{m}=\sum_{i=1}^{m} \lambda_{i} S_{(i)}$ and $Z_{n}=\sum_{j=n}^{\infty} \mu_{j} S_{(j)}$ with $\lambda_{i}, \mu_{j}$ real. For this condition implies $\max _{Y}^{\prime}{ }_{m}^{\prime} Z_{n}^{\prime} \operatorname{cov}\left(Y_{m}^{\prime}, Z_{n}^{\prime}\right) \rightarrow 0$ where $Y_{m}^{\prime} \in \mathbb{B}\left(S_{(1)} \cdots S_{(m)}\right)$ and $Z_{n}^{\prime} \in \mathbb{B}\left(S_{(n)}, S_{(n+1)}, \cdots\right)$, (see $[13$, p. 134]), and this latter condition of asymptotic separation of Borel fields implies $\mathfrak{T}=0$ (see [15]).

Of course, one can obtain less interesting rules $(n)$ with $\mathfrak{T}() \neq 0$, e.g., by having $1 \in(2 n+1), 1 \notin(2 n)$ so that $X_{1}=S_{(2 n+1)}-S_{(2 n)} \in \mathbb{B}\left(S_{2 n}, \ldots\right)$ for all $n$. Similarly, we can algebraically arrange that $\mathfrak{T}()=\mathbb{B}\left(X_{1}, X_{2}, \ldots\right)$ by having each $j \in(n)$, and $\notin(m)$ for arbitrarily large $m, n$.

The rules $S_{\langle n\rangle}$ and $S_{\langle n-1\rangle}+Y$ are intuitively close. However, since their tail $\sigma$-fields are different, the respective measures they induce on their sample spaces are not equivalent and are thus orthogonal since we are dealing with normal processes [17]. It is also possible to find a rule intuitively close to $S_{n}$ which has a trivial tail $\sigma$-field like $S_{n}$ but induces a measure orthogonal to that induced by $S_{n}$. Such an example is given by $S_{(n)}=S_{n-1}+Y_{n}$ where $\left\{Y_{n}\right\}$ is a sequence of independent random variables all distributed as $X_{1}$ and independent of the sequence $X_{j}$. The orthogonality of $S_{(n)}$ and $S_{n}$ is shown by a standard computation with the Hellinger integral [14].

V. More general normal processes.

Theorem 1N. Let $Y_{n}$ be a centered normal process:

(a) $\forall j \leq n, 0 \leq E\left(Y_{n}^{n} Y_{j}\right) \leq E\left(Y_{j}^{2}\right)=\sigma_{j}^{2}$.

(b) $\forall j \leq n, \sigma_{n}^{2}-\sigma_{j}^{2} \geq \sigma_{n-j}^{2}$.

(c) $\sum_{j=1}^{N}\left(1 / \sigma_{j}\right) \rightarrow \infty$.

Then $\forall a \in R, \forall \epsilon>0, \forall \delta>0$

$$
\begin{gathered}
\lim _{n \rightarrow \infty} \frac{1}{\lg \left(\sum_{i=1}^{n}\left(1 / \sigma_{j}\right)\right)} \sum_{k=1}^{n} \frac{1}{\sum_{i=1}^{k}\left(1 / \sigma_{j}\right)} \phi\left[\left|Y_{k}-a\right|<\epsilon\right] \rightarrow \sqrt{2 / \pi} \epsilon \text { a.e. } \\
P\left[\left|\sum_{k=1}^{n} \phi\left[\left|Y_{k}\right|<\epsilon\right]-\sum_{k=1}^{n} \phi\left[\left|Y_{k}-a\right|<\epsilon\right]\right|\right. \\
\left.>(\lg n)^{1+\delta}\left(\sum_{i=1}^{n}\left(1 / \sigma_{i}\right)\right)^{\delta+1 / 2} \text { i.o. }\right]=0,
\end{gathered}
$$




$$
\sum_{k=1}^{n} \phi\left[\left|Y_{k}\right|<\epsilon\right] / \sum_{k=1}^{n} \phi\left[\left|Y_{k}-a\right|<\epsilon\right] \rightarrow 1 \text { a.e. }
$$

We note that in typical cases such as $\sigma_{j} \sim \sqrt{j}, \sum_{j=1}^{n} 1 / \sigma_{j} \sim 1 / 2 \sqrt{j}$, so that if $0<\delta<\delta^{\prime},(\lg n)^{1+\delta}\left(\sum_{j=1}^{n}\left(1 / \sigma_{j}\right)\right)^{\delta+1 / 2}<\left[\sum_{j=1}^{n}\left(1 / \sigma_{j}\right)\right]^{\delta^{\prime}+1 / 2}$ for all large $n$, and so the $(\lg n)^{1+\delta}$ factor can be suppressed in the statement of (2).

Corollary 2N. Under bypotheses (a) and (b) of Theorem 1N $\forall a$ P[a is an accumulation point of $\left.Y_{n}\right]={ }_{0}^{1}$ iff $\sum_{j=1}^{n}\left(1 / \sigma_{j}\right)<\infty$ <

In order to prove Theorem $1 \mathrm{~N}$ and its associated Corollary $2 \mathrm{~N}$, we will need analogs of (R2), (R3).

Under hypotheses (a) and (b) of Theorem 1N,

(R2N) $P\left[\left|Y_{n}-a\right|<\epsilon|,| Y_{j}-a \mid<\epsilon\right] \leq P\left[\left|Y_{n-j}\right|<\epsilon\right] P\left[\left|Y_{j}-a\right|<\epsilon\right]$,

(R3N) $P\left[\left|Y_{n}\right|<\epsilon,\left|Y_{j}-a\right|<\epsilon\right]-P\left[\left|Y_{n}-b\right|<\epsilon,\left|Y_{j}-a\right|<\epsilon\right] \leq K\left(1 /\left(\sigma_{n-j}^{3} \sigma_{j}\right)\right)$

for $n>j$.

Proof of (R2N). Let $Y_{j}^{\prime}, Y_{n}^{\prime}$ be jointly normal centered variables with covariance matrix

$$
\left(\begin{array}{rr}
\sigma_{j}^{2} & \sigma_{j}^{2} \\
\sigma_{i}^{2} & \sigma_{n}^{2}
\end{array}\right)
$$

Thus $Y_{n}^{\prime}=Y_{j}^{\prime}+\left(Y_{n}^{\prime}-Y_{j}^{\prime}\right)$ where $\left(Y_{n}^{\prime}-Y_{j}^{\prime}\right)$ is orthogonal to and thus independent of $Y_{j}^{\prime}$. By the proof given in $\$ 3$ of Proposition 2 stated in the introduction

$$
\begin{aligned}
& P\left[\left|Y_{j}-a\right|<\epsilon,\left|Y_{n}-a\right|<\epsilon\right] \\
& \leq P\left[\left|Y_{j}^{\prime}-a\right|<\epsilon,\left|Y_{n}^{\prime}-a\right|<\epsilon\right]=\int_{a-\epsilon}^{a+\epsilon} F_{Y_{n}^{\prime}-Y_{j}^{\prime}}(a-y-\epsilon, a-y+\epsilon) d F_{Y_{j}^{\prime}}(y) \\
& \leq F_{Y_{n}^{\prime}-Y_{j}^{\prime}}(-\epsilon, \epsilon) F_{Y_{j}^{\prime}}(a-\epsilon, a+\epsilon) \leq P\left[\left|Y_{n-i}\right|<\epsilon\right] P\left[\left|Y_{j}-a\right|<\epsilon\right]
\end{aligned}
$$

since $\sigma_{n-j}^{2} \leq \sigma_{n}^{2}-\sigma_{j}^{2}$ by hypothesis.

Proof of (R3N). $Y_{n}=\left(E\left(Y_{j} Y_{n}\right) / \sigma_{j}^{2}\right) Y_{j}+\left[Y_{n}-\left(E\left(Y_{j} Y_{n}\right) / \sigma_{j}^{2}\right) Y_{j}\right] \equiv Z_{n j}+W_{n j}$ We note $E\left(Z_{n j} W_{n j}\right)=0$ and so since $Z_{n j}$ and $W_{n j}$ are jointly normal, they are independent. $d_{n j}^{2} \equiv E\left(Y_{j} Y_{n}\right) / \sigma_{j}^{2} \leq 1$ (by hypothesis). 


$$
\begin{aligned}
Q & \equiv\left|P\left[\left|Y_{n}-b\right|<\epsilon,\left|Y_{j}-a\right|<\epsilon\right]-P\left[\left|Y_{n}\right|<\epsilon,\left|Y_{j}-a\right|<\epsilon\right]\right| \\
& =\left|\int_{(a-\epsilon) d_{n j}^{2}}^{(a+\epsilon) d_{n j}^{2}}\left[F_{W_{n j}}(b-\epsilon-z, b+\epsilon-z)-F_{W_{n j}}(-\epsilon-z, \epsilon-z)\right] d F_{Z_{n j}}(z)\right| \\
& \leq \frac{1}{2 \pi}\left(\max _{R} \frac{1}{w_{n j}} e^{-x^{2} / 2 w_{n j}^{2}}-\min _{R} \frac{1}{w_{n j}} e^{-x^{2} / 2 w_{n i}^{2}}\right) 2 \epsilon \frac{2 \epsilon}{\sigma_{j}}
\end{aligned}
$$

where $w_{n j}^{2}=\operatorname{var} W_{n j}=\sigma_{n}^{2}-\operatorname{var} Z_{n j}=\sigma_{n}^{2}-E^{2}\left(Y_{j} Y_{n}\right) \sigma_{j}^{2} / \sigma_{j}^{4} \geq \sigma_{n}^{2}-\sigma_{j}^{2} \geq \sigma_{n-j}^{2}$ and $R=\left[-\left.\left|a^{n j}-\right| b|-2 \epsilon| a\right|^{n}+,|b|+2 \epsilon\right]$. Since for $\lambda \geq 0,1-e^{-\lambda} \leq \lambda, Q \leq$ $(|a|+|b|+2 \epsilon)^{2}\left(\epsilon^{2} / \pi\right)\left(\sigma_{n-j}^{3} \sigma_{j}\right)^{-1}$

We now mimic the proof of (2) of Theorem 1 to prove (2) of Theorem $1 \mathrm{~N}$. Let $D(l, n) \equiv \sum_{k=l}^{n} \phi\left[\left|Y_{k}-a\right|<\epsilon\right]-\sum_{k=l}^{n} \phi\left[\left|Y_{k}-a\right|<\epsilon\right]$ with $D_{n} \equiv D(1, n)$, and let $M_{l, n} \equiv \sum_{i=l}^{n} P\left[Y_{i} \in(-\epsilon, \epsilon)\right]$. By (R3N),

$$
\begin{aligned}
& E\left[D^{2}(l, n)\right]= O\left(M_{l, n}\right) \\
&+2 \sum_{l \leq j<k \leq n} \sum_{j}\{ P\left[\left|Y_{k}-a\right|<\epsilon,\left|Y_{j}-a\right|<\epsilon\right] \\
&\left.-P\left[\left|Y_{k}-a^{\prime}\right|<\epsilon,\left|Y_{j}-a\right|<\epsilon\right]\right) \\
&+\left(P\left[\left|Y_{k}-a^{\prime}\right|<\epsilon,\left|Y_{i}-a^{\prime}\right|<\epsilon\right]\right. \\
&\left.\left.-P\left[\left|Y_{k}-a\right|<\epsilon,\left|Y_{j}-a^{\prime}\right|<\epsilon\right]\right)\right\} \\
&=O\left(M_{l, n}\right)+O\left(\sum_{l \leq j<k \leq n,} \sum_{k-j} \frac{1}{\sigma_{k}^{3}} \frac{1}{\sigma_{j}}\right)=O\left(\sum_{j=l}^{n} \frac{1}{\sigma_{j}}\right)
\end{aligned}
$$

since $\sigma_{n}^{2} \geq n \sigma_{1}^{2}$ by hypothesis. We now follow the proof of (2) of Theorem 1 with the modifications $\mu_{i}=\left(1 / \sigma_{i}\right)$ in the application of the theorem from Billingsley [2] and $\left(\sum_{j=1}^{n} 1 / \sigma_{j}\right)\left(\log ^{2} 4 n\right)$ replacing $M(n)^{3 / 2+\epsilon+\epsilon "}$ from the part dealing with $\sim k^{\lambda}$ till the end of the proof.

The proofs of (1) and (3) of Theorem $1 \mathrm{~N}$ go through as previously.

We wish to express our gratitude to the referee for his many helpful suggestions especially in formulating the present version of Theorem 4.

\section{REFERENCES}

1. Leonard E. Baum, M. Katz and H. H. Stratton, Strong laws for ruled sums, Ann. Math. Statist. 42 (1971), 625-629.

2. Patrick Billingsley, Convergence of probability measures, Wiley, New York, 1968. MR 38 \#1718.

3. Leo Breiman, Probability, Addison-Wesley, Reading, Mass., 1968. MR 37 \#4841. 
4. K. L. Chung and P. Erdös, Probability limit theorems assuming only the first moment. I, Mem. Amer. Math. Soc. No. 6 (1951). MR 12, 722.

5. K. L. Chung and W. H. J. Fuchs, On the distribution of values of sums of random variables, Mem. Amer. Math. Soc. No. 6 (1951). MR 12, 722.

6. William Feller, An introduction to probability theory and its applications. Vol. I, 2nd ed., Wiley, New York, 1966. MR 42 \#5292; Vol. 2, Wiley, New York, 1966. MR 35 $\# 1048$.

7. Jean-Pierre Kahane, Some random series of functions, Heath Math. Monographs, Raythe on Education, Lexington, Mass., 1968. MR 40 \#8095.

8. Harry Kesten, The limit points of a normalized random walk, Ann. Math. Statist. 41 (1970), 1173-1205. MR $42 \# 1222$.

9. Simon Kochen and Charles Stone, $A$ note on the Borel-Cantelli lemma, Illinois J. Math. 8 (1964), 248-251. MR $28 \# 4562$.

10. B. V. Gnedenko and A. N. Kolmogorov, Limit distributions for sums of independent random variables, GITTL, Moscow, 1949; English rev. transl., Addison-Wesley, Reading, Mass., 1968. MR 12, 839; MR 38 \#1722.

11. M. Katz, The probability in the tail of a distribution, Ann. Math. Statist. 34 (1964), 312-318.

12. M. Loeve, Probability theory, 3rd. ed., Van Nostrand, Princeton, N. J., 1963. MR $34 \# 3596$.

13. Ju. V. Prohorov and Ju. A. Rozanov, Probability the ory: Basic concepts, limit theorems, random processes, "Nauka", Moscow, 1967; English transl., Die Grundlehren der math. Wissenschaften, Band 157, Springer-Verlag, New York, 1969. MR 36 \#2175; MR $40 \# 4981$.

14. C. R. Rao and V.S. Varadarajan, Discrimination of Gaussian processes, Sankhya Ser. A 25 (1963), 303-330. MR $32 \# 5 ̣ 72$.

15. P. Revesz and P. Bartfai, On a zero-one law, Z. Wahrscheinlichkeitsthe orie und Verw. Gebiete 7 (1967), 43-47. MR 34 \#8454.

16. Herbert $\mathrm{E}$. Robbins, On the equidistribution of sum of independent random variables, Proc. Amer. Math. Soc. 4 (1953), 786-799. MR 15, 139.

17. L. A. She pp, The singularities of Gaussian measures in function space, Proc. Nat. Acad. Sci. U.S.A. 52 (1964), 430-433. MR $29 \# 6536$.

18. D. Slpeian, The one sided barrier problem for Gaussian noise, Bell System Tech. J. 41 (1962), 463-501. MR 24 \#A3017.

19. Charles Stone, A local limit theorem for non lattice multidimensional distribution functions, Ann. Math. Statist, 36 (1965), 546-551. MR 30 \#5351.

20. H. Weyl, Über die Gleichverteilung von Zahlen mod Eins, Math. Ann 77 (1916), 313-352.

INSTITUTE FOR DEFENSE ANALYSES, PRINCETON, NEW JERSEY 08540 